\title{
1 Non-destructive internal disorder detection of Conference 2 pears by semantic segmentation of X-ray CT scans using 3 Deep Learning
}

4 Tim Van De Looverbosch 1,a, Ellen Raeymaekers ${ }^{1, b}$, Pieter Verboven ${ }^{1, c}$, Jan Sijbers ${ }^{2, d}$, Bart Nicolai ${ }^{1,3, e}$

$5{ }^{1}$ Mechatronics, Biostatistics and Sensors (MeBioS), Biosystems Department, KU Leuven, Belgium

62 imec Vision Lab, Department of Physics, University of Antwerp, Belgium

$7 \quad 3$ Flanders Centre of Postharvest Biology, Belgium

9 a tim.vandelooverbosch@kuleuven.be; b ellen.raeymaekers@student.kuleuven.be;

c pieter.verboven@kuleuven.be; d jan.sijbers@uantwerpen.be; ${ }^{e}$ bart.nicolai@kuleuven.be

\section{Abstract}

Long term storage is required to deliver high quality pear fruit year-round. Under suboptimal storage conditions, internal disorders, such as internal browning and cavity formation, can develop and are often invisible from the outside. We present a non-destructive inspection method to quantify internal disorders in X-ray CT scans of pear fruit using a deep neural network for semantic segmentation. Herein, a U-net based model was trained to automatically indicate healthy tissue, core and regions affected by internal disorders, i.e., cavity formation and internal browning. The quantitative data resulting from the segmentations was used to measure the severity of internal disorders. Excellent classification accuracies of 99.4 and $92.2 \%$ were obtained for the classification of "consumable" vs "non-consumable" fruit on the one hand and "healthy" vs "defect but consumable" vs "non-consumable" fruit on the other hand. The identification of "defect but consumable" fruit showed to be the most difficult.

Keywords:

Postharvest Technology; Food Quality Inspection; Fresh Commodity Sorting; Computed

\section{Introduction}

There is an increasing worldwide demand for high quality pome (apple and pear) fruit yearround. Since prome fruit is only harvested once a year, the fruit must be stored for a long time with minimal quality decay (Mercier et al., 2017). Unfortunately, internal disorders, such as internal browning and cavity formation, can develop under suboptimal storage conditions (Franck et al., 2007; Lammertyn et al., 2000; Pedreschi et al., 2009; Veltman et al., 2003). The affected fruit often shows no external symptoms. Consequently, the internal quality cannot be 
evaluated at the time of purchase but influences the repurchase decision of consumers. Severe internal disorders make the fruit thus unmarketable and defective fruit reaching retail and endcustomers should be avoided.

With current commercial quality grading systems, internal disorders cannot to be detected reliably (Huang et al., 2020; Nicolaï et al., 2014). False negatives adversely impact the repurchase decision, while false positives can lead to a significant financial loss for the growers and fruit auction cooperatives. For this reason, fast and inline non-destructive methods to inspect the internal quality of individual products have been investigated (Han et al., 2006; Huang et al., 2020; Khatiwada et al., 2016; Kotwaliwale et al., 2014; van Dael et al., 2018, 2017). However, many of those methods are focussed on purely classifying healthy and defective fruit. Jaeger et al. (2016) showed that a certain percentage of internal defects can be acceptable to consumers, e.g. little cavities in the fruit are less problematic than large patches affected by internal browning. It seems, therefore, beneficial to be able to quantify the severity of certain disorders to be able to make more informed decisions. With binary classification systems, it is clearly impossible to obtain such fine information and make nuanced decisions.

A promising technology for non-destructive internal quality inspection is X-ray computed tomography (CT), since spatial information on density differences can be provided in 3D (Chigwaya et al., 2018; Diels et al., 2017; Herremans et al., 2013, 2014; Lammertyn et al., 2003b, 2003a; Van De Looverbosch et al., 2020). Significant progress has been made towards inline Xray CT applications of food inspection (De Schryver et al., 2016; Janssens et al., 2016, 2018; Pereira et al., 2017), with one of the main challenges remaining automatic image analysis. Recent work by our group has shown that automated feature extraction on X-ray CT data of pear fruit followed by a classification by means of a support vector machine (SVM), is effective in classifying fruit based on the presence of internal disorders (Van De Looverbosch et al., 2020). However, this approach did not allow the quantification of the severity of the internal disorders. Moreover, the used features had to be hand-crafted and finding the right features required considerable trial and error. Automatic and accurate segmentation of the internal disorders would enable the quantification of their severity and potentially improve classification results. However, due to the large biological variability, the development of such algorithms using classical image processing and machine learning approaches is challenging. Fortunately, deep learning has recently become a viable tool for pattern recognition and image interpretation tasks and was found to be successful in tasks using medical X-ray CT data (Goodfellow et al., 2016; LeCun et al., 2015; Litjens et al., 2017; Shen et al., 2017).

The aim of this study is two-fold. First, a non-destructive quality inspection method is developed to quantify internal disorders in pear fruit based on X-ray CT scans. Hereto, a deep neural network 
for semantic segmentation (U-net) is trained to indicate various structures and tissues, including internal disorders, in the X-ray CT scans. Second, it is investigated if the quantitative data can be used to accurately classify the fruit, on the one hand, in "consumable" or "non-consumable" categories and, on the other hand, into "healthy", "defect but consumable" or "non-consumable" categories. Herein, ground truth classifications were obtained by a survey with images of cut fruit, in which participants indicated if they could spot internal disorders ("defect") or not ("healthy") and whether they would eat the fruit ("consumable") or not ("non-consumable").

\section{Materials and methods}

The materials and methods are discussed in detail in the following sections. In Figure 1, a flowchart is presented to provide a general overview of the materials and methods.

\subsection{Pear fruit and storage protocols}

A Flemish grower (5053'06.4" N 501'32.2" E, Kortenaken, Belgium) harvested and delivered $160 \mathrm{~kg}$ of Conference pears on August 24, 2018. The fruits were randomly divided in two groups and put in long term storage for eight months in controlled atmosphere (CA) storage facilities of the Flanders Centre of Postharvest Technology (VCBT, Leuven, Belgium). For the first 25 days both groups were put at $-1{ }^{\circ} \mathrm{C}$ in normal atmosphere to acclimatize to the cold temperature, after which different CA conditions for each group were implemented in CA containers with a volume of $1 \mathrm{~m}^{3}$. The first group received the control optimal storage treatment following the recommendations of the VCBT for commercial sale, with a temperature, $\mathrm{O}_{2}$ partial pressure and $\mathrm{CO}_{2}$ partial pressure of $-1{ }^{\circ} \mathrm{C}, 2.5 \mathrm{kPa}$ and $0.7 \mathrm{kPa}$, respectively (VCBT, 2017). For the second group, experimental conditions were implemented to induce internal disorder development with a temperature, $\mathrm{O}_{2}$ partial pressure and $\mathrm{CO}_{2}$ partial pressure of respectively $-1{ }^{\circ} \mathrm{C}, 1.0 \mathrm{kPa}$ and $4.0 \mathrm{kPa}$. The fruits were removed from CA storage on May 9, 2019. 90 fruits of each treatment were selected randomly and stored at $-1^{\circ} \mathrm{C}$ in normal atmosphere before further measurements.

\subsection{X-ray CT scans and data pre-processing}

Of each treatment, 90 samples were randomly selected. The fruits were then divided into groups of thirty over six plastic boxes in which they were separated by styrofoam grids. The grids divided each box into two layers of fifteen samples and facilitated image processing afterwards by guaranteeing fruit were not touching each other. Next, the boxes were carefully brought to the UZ Leuven hospital in Leuven (Belgium) for obtaining X-ray CT images with a gantry CT system (SOMATOM Definition Flash, Siemens, Germany). Three boxes could be imaged at once. The system operated at $100 \mathrm{kVp}$ with a voxel size of $0.9766 \times 0.9766 \times 0.3000 \mathrm{~mm}^{3}$. 

scan of one box (Figure 2a, b and c) and illustrates some of the performed image processing steps.

102 First, the volumes were resampled to a $0.9766 \times 0.9766 \times 0.9766 \mathrm{~mm}^{3}$ voxel size. Individual fruits were then cropped (Figure 2d) automatically and identified based on their position to link their CT images to their reference images (see section 2.3). The background of the CT volumes was removed with a global Otsu threshold (Figure 2e) (Otsu, 1979) and intensity values of the CT volumes were scaled between $[0,1]$ by dividing the intensities by the maximum value encountered over all volumes. Since fruit position was not fixed in the boxes, pears in the 3D volumes had to be aligned to the same pose. Therefore, each sample was centralized and rotated so that their principal axis aligned with the Z-axis and the fruit was in upwards orientation. To ensure upwards orientation, the fruits were rotated $180^{\circ}$ over the $\mathrm{x}$-axis if the center of mass was in the top half of the volume (Figure 2f). Finally, all volumes were padded with background voxels to the maximum size in the X-, Y- and Z-dimension encountered over all 180 volumes, resulting in identical volumes of $184 \times 179 \times 198$ voxels.

\subsection{Reference images and ground truth classification}

Following the CT scans, the fruits were consecutively cut open and reference images of the fruit flesh were taken. Multiple slices were cut perpendicular to the style-calyx axis and spread out below an RGB-camera (see Figure 3). Afterwards, an online survey was organized with the reference images in which the participants were asked to indicate on the one hand whether they found the fruit to be healthy or defective and on the other hand if they considered the fruit consumable or not. The survey was sent out to everyone working for the research group and completed by 17 participants. A majority vote was implemented over all participants to decide on the ground truth classification of each fruit ("healthy" vs. "defect" and "consumable" vs. "nonconsumable"). Herein, the strength of the inter-annotator agreement, as measured by Fleiss' kappa statistic, was found to be moderate (0.52) (Fleiss, 1971; Landis \& Koch, 1977).This resulted in a dataset of 126, 26 and 26 samples labelled as "healthy", "defect but consumable" and "non-consumable", respectively.

\subsection{Manual ground truth annotation}

128 CT volumes of 51 out of the 180 pears were manually annotated using the free and open-source 3D Slicer software (3D Slicer, 2020; Fedorov et al., 2012). Prior to manual annotation of each sample, the cut open reference image of each fruit was examined for the presence and severity of internal disorders. In the segmentation software, voxels were given one of five different classes:

132 "external air", "healthy tissue", "core", "cavity" and "internal browning". First, "external air" was 133 indicated by applying a global Otsu-threshold and preserving the largest island, i.e., the largest connected component. Secondly, the other volumes with a value below the Otsu-threshold inside 
the fruit were assigned to the "core" or "cavity" class. Hereby, the "core" island was manually indicated. Third, between 5 and 10 seed points were manually placed in regions of "healthy tissue" and regions affected by "internal browning". Then, the region growing algorithm in 3D Slicer ("Grow from seeds") was used to complete the annotation. Herein, the other classes were fixed. Finally, a smoothing operation was done on the "healthy tissue" and "internal browning" regions using the "Joint smoothing" method in 3D slicer with a smoothing factor of 0.50 . Figure 4 shows the steps in the manual annotation procedure of the CT volumes.

\subsection{Dataset generation}

The YZ slices (179 x 198 pixels) of the pre-processed CT and corresponding manually annotated volumes were used to train the U-net segmentation model. For the input of the model, three consecutive slices were concatenated in the third dimension, resulting in an image with three channels. As target, the manually annotated slice of the middle of the three input slices was used. The slices adjacent to the middle input slice are thus used to to provide contextual information. The slices of the 51 annotated volumes were divided in training, validation and test sets. Hereof, slices with only external air were omitted since they are not of interest. The slices of 36 samples were used for training and validation, including 14 "healthy", 10 "defect but consumable" and 12 "non-consumable" samples. $10 \%$ of the slices were used for validation during the training process. The training and validation set included in total 2172 and 241 slices, respectively. Finally, the test set included the slices of 5 "healthy", 5 "defect but consumable" and 5 "nonconsumable" samples with 2760 slices in total.

\subsection{Network architecture and training for segmentation}

A U-Net based network of four downsampling and four upsampling blocks was adapted to learn the multi-class semantic segmentation of regions of interest ("external air", "healthy tissue", "core", "cavity" and "internal browning" pixels) in the YZ X-ray CT slices (Milesial, 2019; Ronneberger et al., 2015). The model architecture is presented in Figure 5. The input layer had three channels of $179 \times 198$ pixels. After each 3x3 convolution, batch normalization and the ReLU activation function was applied (Ioffe \& Szegedy, 2015; Nair \& Hinton, 2010). In the downsampling steps, max pooling with a $2 \times 2$ kernel was used. Upsampling was done using a scale factor of 2 and bilinear interpolation. Skip connections were used to concatenate the output of each downsampling block to the output of the corresponding upsampling block. Herein, the output of the upsampling block was padded with zeros to match the height and width of the output of the downsampling block. The output layer had five channels of the same size, one for each class. The segmented image (only one channel) was then obtained by assigning each pixel to the class with the largest probability in the output layer. 
The model was implemented in python using the PyTorch framework version 1.4.0 (Paszke et al., 2019) and trained for 20 epochs, i.e. for 20 passes over the training set. As loss function, i.e. the function used to calculate the error between the model's prediction and the ground truth, the class-weighted cross-entropy loss function was used to account for class imbalance in the data (Sudre et al., 2017). The used class-weights were determined based on the ground truth data in the training set. Hereto, the differences between the total number of voxels and the number of voxels belonging to each class were first calculated. Then, the ratio of these differences and the total number of voxels was used as class-weight, resulting in the following weights: 0.08 ("external air"), 0.93 ("healthy tissue"), 1.00 ("core"), 1.00 ("cavity") and 0.99 ("internal browning"). The performance on the validation set was tested after every epoch and was used to optimize the batch size and initial learning rate hyper-parameters. The batch size is the number of samples the model handles during one iteration. During training, the weights of the model were updated after each iteration proportional to the loss on each batch. The magnitude of the weight changes depended on the learning rate, which started from the initial learning rate set by the operator. Here, the Adam optimizer was used to dynamically change the learning rate during training (Kingma \& $\mathrm{Ba}, 2014$ ). The effect of data augmentation in the form of affine image transformations (random mirroring, rotating, shearing and scaling) on the training data was tested.

\subsection{Segmentation performance validation}

After model training was finished, the segmentation performance was validated by comparing the predictions with the ground truth annotation using the Intersection-Over-Union metric (IoU, also known as the Jaccard index) (Rezatofighi et al., 2019). The IoU is a common metric to evaluate the correspondence between two shapes and has a range between 0 (no correspondence between prediction and ground truth) and 1 (full correspondence between prediction and ground truth). It was calculated for each label on slice level for the test set. The IoU for a certain label was not calculated for slices in which the label was not present in the prediction or ground truth. The mean IoU was calculated per pear and the overall mean and box plot for the IoU of each label were calculated. Finally, the mean and box plots were also calculated for the slices of "healthy", "defect but consumable" and "non-consumable" samples, separately.

\subsection{Classification}

199 Quantitative data obtained from the output of the trained segmentation model was used to 200 classify the fruit. Therefore, all consecutive slices of all 180 samples were segmented by the U-net model and the percentages of the fruit volumes corresponding to "cavity" and "internal browning" were calculated. The same calculations were performed on the manual ground truth segmentations. First, the accuracy of the predicted percentage of internal browning was 
investigated. Hereto, bins were made of $2 \%$ in size, resulting in the bins $[0.0-2.0 \%, 2.0-4.0 \%, \ldots$, 98-100 \%] and it was tested if the prediction fell into the same bin as the ground truth. Second, a binary classifier, a logistic regression model (Kutner et al., 2005), was fitted using a 5-fold crossvalidation to separate "consumable" and "non-consumable" samples based on the quantitative data of cavity and brown volume fraction. Finally, a multiclass classifier, a quadratic discriminant model (Tharwat, 2016), was fitted on the same features using a 5-fold cross-validation to separate "healthy", "defect but consumable" and "non-consumable" samples. The classification results of both classifiers are presented using confusion matrices and receiver operating characteristic (ROC) curves (Fawcett, 2006; Metz, 1978).

\section{Results}

\section{$214 \quad 3.1$ Segmentation model training}

215 The best results were obtained without data augmentation and with a batch size and initial 216 learning rate of 4 and $1.0^{-4}$, respectively. Final training and validation losses of, respectively, 0.004 217 and 0.011 were achieved. The training and validation loss during training is presented in Figure

218 6. Interestingly, data augmentation did not improve the results (final validation loss of 0.016). 219 The remainder of this section therefore discusses the results obtained without data augmentation.

221 Figure 7 shows a CT slice, its ground truth segmentation, its predicted segmentation and reference image of five samples of the test set. In the first row, a slice of a "defective" pear is shown in which the core and a large cavity were connected, i.e., the core and cavity were indistinguishable. Therefore, no core was labelled during the ground truth annotation, while only cavity is indicated in the prediction. The second row shows a slice of a "healthy" pear that was correctly segmented. On the third row, the results on a slice of a "defect" pear with cavities and browning is shown. The ground truth and prediction correspond quite well. Finally, in the fourth row a slice of another "defect" pear is shown with good similarity between the ground truth and prediction.

\subsection{Segmentation performance validation}

231 In Figure 8, the boxplots of the mean IoU scores, calculated per pear of the test set, are shown for the different label categories. For "external", "healthy tissue" and "core" high mean IoU scores were obtained, meaning there is a good agreement between the ground truth and predicted segmentation. For "core", also outliers with a lower score are present. The median IoU for "cavity" is still quite high (0.95), but the interquartile range is greater, meaning there is a larger spread in the scores. For "internal browning", a median and mean IoU of 0.0 and 0.2 were found. The low 
score of 0.0. In case of IoU score of 0.0, no overlap was found between the ground truth and the prediction. It can also indicate the presence of a certain label in the prediction, which is not present in the ground truth (or vice versa).

Figure 9 shows IoU scores calculated on individual slices of the three different categories of pear ("healthy", "defect but consumable" and "non-consumable") for the test set. For "internal browning", the IoU scores are equal to 0.0 for both the categories "healthy" and "defect but consumable": there is actually no or very little browning present in these pears. For the "nonconsumable" category, however, the median is equal to 0.61 and the IoU scores are overall higher. This shows that due to the low IoU scores of the first two categories the overall IoU score of "internal browning" is low. In the plot the first category, high IoU scores are present for "external", "healthy tissue" and "core", which is desirable since this category contains healthy fruit. There is, however, also an IoU score of 0.0 for "cavity" present, meaning an incorrect prediction of cavity. For the other categories, also the first three labels exhibit high scores. For "cavity", the median is high, but outliers are present with lower scores.

\subsection{Classification}

253 Based on the majority vote on survey results, all 180 samples were assigned to a ground truth category. 128, 26 and 26 samples were found to be "healthy", "defect but consumable" and "nonconsumable", respectively. Thereafter, the trained U-net model was used to segment all slices of each fruit and the percentage of "cavity" and "internal browning" was calculated. Subsequently, the predicted percentages of "internal browning" were compared to the percentages obtained from the ground truth segmentation of the test set by testing if the predicted percentages fell into the same bins as the ground truth ([0.0-2.0 \%, 2.0-4.0 \%, ..., 98-100 \%]) (see section 2.8). Figure 10 shows a histogram of the predicted and ground truth percentages of "internal browning" for the test set. The prediction of only two samples of the test set was put in the wrong, but adjacent, bin. Note that the test set included samples with internal browning up to around $15 \%$. However, it was found that for the training set, with internal browning percentages going up to around $45 \%$, the predicted and ground truth bin were in disagreement for only three samples (results not shown).

266 Thereafter, a binary ("healthy" and "defective") and a multiclass ("healthy", "defect but consumable" and "non-consumable") classifier were fitted to the predicted percentages of "internal browning" and "cavity" data of all 180 fruit in a 5-fold cross-validation. Figure 11 shows the scatterplots of the dataset with ground truth categories for the binary and multiclass classification. 
The confusion matrices of the binary (logistic regression model) and multiclass classifiers (quadratic discriminant model) are shown in Table 1 and Table 2. The binary classifier achieved an overall accuracy, true positive and true negative rate of $99.4,96.0$ and $100.0 \%$ in classifying "consumable" and "non-consumable" samples. The multiclass classifier achieved an overall accuracy of $92.2 \%$ and true positive rates of $97.0,65.0$ and $96.0 \%$ for "healthy", "defect \& consumable" and "defect \& non-consumable", respectively. While high accuracies were thus obtained for the "healthy" and "defect \& non-consumable" categories, a substantial part of the "defect \& consumable" category was misclassified.

Since the binary classifier obtained a true positive and false positive rate of respectively 96.0 and $0.0 \%$, no ROC-curve is shown for this classifier (Area Under the Curve (AUC) $=1$ ). Figure 12 shows the ROC curves of the multiclass classifier. Herein, the ROC-curves are presented per category using a one-vs-all approach, i.e. the ROC-curves are shown for each category with the respective category as the positive class and the others combined as the negative class. The AUC were equal to $0.93,0.87$ and 1.00 for respectively, "healthy", "defect \& consumable" and "defect \& non-consumable" as positive class.

\section{Discussion}

\subsection{An efficient and reproducable 3D manual annotation procedure}

The manual annotation of 3D data is relatively difficult, especially when it can only be performed on 2D slices. Therefore, we developed a reproducible procedure that uses 3D operations minimally dependent on subjective factors. The threshold to separate voxels comprising air and tissue was determined objectively using Otsu's method (Otsu, 1979).

The external air, cavities and core could also be objectively determined for most samples. However, some pairs were found "difficult" to annotate when cavities were connected with the core of the fruit (see Figure 13). During the ground truth annotation, the connected core and cavity were respectively labelled as "core" and "cavity" when they could easily be distinguished, e.g., only a small hole connecting both areas. However, in case the core and cavity could not be distinguished anymore, the whole opening was labelled as "cavity". It was found that in these cases, the deep neural network labelled some voxels of the internal space as "core", even though no clear boundary could be identified between the "core" and "cavity". Of course, the way of annotating these samples had an impact on the segmentation metrics for the "cavity" and "core" label.

The annotation of "tissue" and "internal browning" was relatively fast using the semi-automatic seeded region growing algorithm (see Figure $4 b$ and c). The placements and number of the initial 
seeds, however, was done subjectively and different seed points could possibly cause different results. Alternatively, the use of a predetermined threshold to segment these regions would make the process easier. However, this was impossible due to the variability in intensity values of the healthy tissue and internal browning between different samples. (Lammertyn et al., 2003b) used minimally different thresholds on each pear to separate the tissue and internal browning segments, which is, however, a subjective and equally time-consuming approach. Our annotation process was done by one person. Preferably, this should be done by multiple operators. Although reference images of all fruit were present to guide the operator, this could prevent an operatorspecific annotation and improve the performance of the segmentation model.

\subsection{A segmentation model to quantify the internal disorder severity}

A deep neural network was trained to automatically segment different tissue types and structures in slices of X-ray CT scans of pear fruit. Data augmentation in the form of affine transformations on the training data did not improve on the results obtained without data augmentation. In general, data augmentation is useful for preventing neural networks from overfitting on the training data. However, overfitting on the training data was not observed for the model trained without data augmentation (see Figure 6). The effect of data augmentation might thus be insignificant. Additionally, the nature of our dataset possibly makes data augmentation less effective. In the preprocessing of the data, all CT reconstructions were put in the same pose (see section 2.2). The preprocessing, which can also be done on new data, makes sure all samples are presented to the model in the same manner. Images resulting from data augmentation might therefore not be encountered in the actual dataset. The model would then generalize to irrelevant cases. It was therefore decided to use the model trained without data augmentation.

Visually, the predicted segmentations of many samples corresponded well with the ground truth annotations (see section 3.1). A validation of the segmentation performance using the IoU metric on an independent test set (see section 3.2) showed high scores for the labels "external", "healthy tissue" and "core". A high median IoU was also obtained for the "cavity" label, but a larger range was found including lower values. Interestingly, low IoU scores were found for the "internal browning" label, even though visually most predictions seemed sufficiently accurate. It turned out this was mainly caused by errors on small volumes and volume edges. Since the IoU metric is relative to the ground truth, the absolute size of the volumes did not matter, resulting in low IoU scores even though the error by the model was rather negligible.

Particularly, it was observed that many low IoU scores for "internal browning" were caused by the incorrect labelling of small pixel regions, or regions forming a boundary around the core or cavities in the predicted image (see the first row in Figure 7). These misclassified regions resulted 
in an IoU of 0.0 if no "internal browning" was present in the ground truth. Typically, these misclassified regions had lower grey scale intensity values similar to the intensity of regions affected by internal browning. The latter had lower intensity values due to a lower density and higher porosity (Nugraha et al., 2019). However, for the experienced eye, supported by the reference images, it was clear that internal browning had often not occurred. Rather, the partial volume effect of the CT imaging was probably responsible for the local decrease in pixel intensity

344 (Barrett \& Keat, 2004). Due to the limited resolution of the CT scans $345\left(0.9766 \times 0.9766 \times 0.9766 \mathrm{~mm}^{3}\right.$ voxel size), voxels in the transition of two regions with different 346 X-ray attenuations (e.g., different densities) have an intensity somewhere in between. The partial 347 volume effect is thus present at the boundary of the core or cavities, resulting in the 348 misclassification of the affected pixels by the network. Moreover, some cavities were too small to 349 be thresholded in the CT scans and were therefore not annotated as "cavity" during ground truth annotation. Nonetheless, these small cavities caused small spots with lower intensity due to the partial volume effect and were often indicated as "internal browning" in the predicted image (see the last row in Figure 7). Even though low IoU scores were obtained for "internal browning", it was shown that the severity of the internal disorders could be accurately predicted, and the misclassified voxels had a relatively small impact on the subsequent classification.

An interesting case was found in which a cavity was predicted to be the core of the fruit (see Figure 14). Indeed, when looking at the CT slice in Figure 14a, the shape and location of the cavity in the slice resembles the typical shape and location of the core. From examining the CT volume, however, it clearly was a cavity and was labeled as such during ground truth annotation (see Figure 14b). Since the model does its predictions slice-by-slice, it lacks the 3D information required to correctly recognize the whole as a cavity. A 3D implementation of the U-Net model could be beneficial to avoid such mistakes (Çiçek et al., 2016). However, to train such model more labelled CT-scans would be required.

Several strategies can be followed to improve the segmentation results and robustness. First, as stated before, a 3D implementation of the segmentation model could be applied so that the model can use 3D information to prevent mistakes as presented in Figure 14. Second, more samples could be manually annotated, including more "difficult" samples. Here, 51 out of the 180 available samples were annotated with a preference for "easier" samples (see sections 2.4 and 4.1). To segment "difficult" samples, one must attempt to design an objective method. Third, data augmentation could be applied on the CT scans and/or CT slices to artificially increase the size and variability of the dataset. Finally, additional datasets could be included of fruit from different years, origins and maturity stages. 
373 The trained deep neural network was used to obtain accurate predictions on the severity of 374 internal disorders, expressed in percentages of the fruit volume affected by a disorder (see Figure 375 10). Based on the quantitative data, classifiers were trained to separate "consumable" and "nonconsumable" fruit on the one hand, and "healthy", "defect but consumable" and "non-consumable" fruit on the other hand. Excellent classification performance was achieved for the former classifier, with an overall accuracy, false positive and false negative rate of $99.4,0.0$ and $4.0 \%$ (see Table 1). "Non-consumable" fruit can thus be reliably separated from sound fruit using our method. For the multiclass classifier, great performance was achieved for "healthy" and "nonconsumable" samples, but a true positive rate of only $65.0 \%$ was achieved for the "defect but consumable" category (see Table 2). It was found that $89.0 \%$ of the misclassified samples of this category were predicted to be "healthy". This could be caused by similar percentages of "cavity" and "internal browning" as "healthy" samples, e.g., only very small internal disorders. This reasoning was confirmed, as all misclassified samples were located in the bottom left corner of Figure 11. Additionally, possibly an insufficient segmentation of the defective regions might have occurred, causing an underestimation of the actual disorder severity. Moreover, it should be noted that the alignment step of the pears during pre-processing included interpolation and resampling procedures that might have altered the appearance of the CT scan. However, the majority of this category could be identified correctly, and misclassifications might not be a big concern since the fruit was still considered consumable.

It was found that some internal defects were visible in the reference images of the cut-open fruit, but not in the CT volumes. Recent bruises, for instance, might manifest themselves in the reference image as regions with free water in the intercellular space. Since the water has not evaporated yet, there might not yet be a difference in density and thus X-ray attenuation. An alternative explanation might be that the bruises only occurred after the CT scans were acquired during transport in the period before the reference images were taken. These defective regions were, therefore, visible in the reference images but could not be segmented in the predication or the ground truth volumes and were, hence, not quantified. Figure 15 shows the reference image and orthogonal slices of a bruised fruit categorized as "defect but consumable". Contrary, some disorders might have been invisible in the reference images due to the positions where the cuts were made, resulting in an incorrect categorization. systems by nature. Here we proposed a two-step approach, in which a semantic segmentation model is used to deliver quantitative data based on which the fruit are subsequently classified. 
is transparent, because the output of the segmentation model that precedes the classification can be visualized and expressed quantitatively. Second, the method can be easily adapted for different markets, as it would only require retraining the classifier on categories specific to the different markets. The segmentation can be assumed independent of the market and retraining the segmentation model would thus not be required. End-to-end trained classifiers, on the other hand, would require retraining for each market. In future work, an end-to-end system could be investigated in which a trained 3D segmentation model is used as an initialization of a model with the same architecture, followed by a small convolutional neural network for final classification. To adapt the model to a certain market, the segmentation layers could be frozen, while the classification layers are retrained with market specific categories. Alternatively, the segmentation and classification could be learned simultaneously, using multi-task learning (Ruder, 2017).

\subsection{Potential applications}

Automatic segmentation of CT-scans can significantly speed up the analysis of internal defects in foods. Various promising applications are conceivable. First, the method could be applied to nondestructively inspect the internal quality foods in a quantitative way and to accurately predict the severity of internal defects. This would not only allow separating non-consumable from consumable products, but also to separate consumable products of different internal quality. Since the absence of internal defects can be guaranteed, higher margins are justifiable. However, significant improvements are needed in hardware and software to implement automated X-ray CT analysis at the throughput of commercial sorting lines (e.g. at least ten products per second for fruit sorting). In this regard, research is done on obtaining high quality reconstructions with a minimal number of projections and inline X-ray CT approaches (De Schryver et al., 2016; Janssens et al., 2016, 2018; Pereira et al., 2017). Second, this method might facilitate more fundamental research on internal defect development on a larger scale, since cumbersome manual analysis can be automated (Lammertyn et al., 2003a; Pedreschi et al., 2009; van Dael et al., 2018). A larger quantity of samples could be included, or the same samples could also be analysed at different time-points during storage with short intervals for CT scanning. Finally, the method could be adapted for foreign object detection in foods (Edwards, 2004; Graves et al., 1998).

\section{Conclusion}

A non-destructive inspection method was developed to quantify internal disorders in pear fruit in X-ray CT scans using a deep neural network for semantic segmentation. Herein, a model was trained to indicate healthy tissue, core and regions affected by internal disorders, i.e., cavity formation and internal browning, in slices of the scans. The severity of internal disorders could 
441 be predicted successfully from the segmentations. Moreover, it was shown that the resulting

442 quantitative data can be used to classify "consumable" vs "non-consumable" fruit at high accuracy 443 on the one hand and "healthy" vs "defect but consumable" vs "non-consumable" classification on 444 the other hand. Herein, the identification of "defect but consumable" fruit showed to be the most 445 difficult. Being able to reliably separate fruit from the three classes would facilitate different 446 market strategies and could justify larger margins on the "healthy" category since high internal 447 quality is guaranteed. The presented method might be of great interest to researchers and 448 industry working on quality assurance, product analysis and foreign object detection in foods and 449 other industries.

\section{Acknowledgements}

451 The financial support of KU Leuven (projects C1/16/002 and C3/19/036), the Fund for Scientific Research (FWO project G060516N and Hercules funding I013518N) and Flanders Innovation and Entrepreneurship (HBC.2016.0806).

\section{References}

3D Slicer. (2020). https://www.slicer.org/

Barrett, J. F., \& Keat, N. (2004). Artifacts in CT: recognition and avoidance. Radiographics, 24(6), $1679-1691$.

Chigwaya, K., Schoeman, L., Fourie, W. J., Crouch, I., Viljoen, D., \& Crouch, E. M. (2018). 'Fuji' apple internal browning explored via X-ray computed tomography (CT). Acta Horticulturae, 1201, 309-316. https://doi.org/10.17660/ActaHortic.2018.1201.42

Çiçek, Ö., Abdulkadir, A., Lienkamp, S. S., Brox, T., \& Ronneberger, O. (2016). 3D U-Net: Learning Dense Volumetric Segmentation from Sparse Annotation. http://arxiv.org/abs/1606.06650

De Schryver, T., Dhaene, J., Dierick, M., Boone, M. N., Janssens, E., Sijbers, J., Dael, M. V., Verboven, P., \& Nicolai, B. (2016). In-line NDT with X-Ray CT combining sample rotation and translation. NDT and E International, 84, 89-98. https://doi.org/10.1016/j.ndteint.2016.09.001

Diels, E., van Dael, M., Keresztes, J., Vanmaercke, S., Verboven, P., Nicolai, B., Saeys, W., Ramon, H., \& Smeets, B. (2017). Assessment of bruise volumes in apples using X-ray computed tomography. Postharvest Biology and Technology, 128, 24-32. https://doi.org/10.1016/j.postharvbio.2017.01.013 
Edwards, M. (2004). Detecting foreign bodies in food. Elsevier.

471 Fawcett, T. (2006). An introduction to ROC analysis. Pattern Recognition Letters, 27(8), 861-874. https://doi.org/10.1016/j.patrec.2005.10.010

Fedorov, A., Beichel, R., Kalpathy-Cramer, J., Finet, J., Fillion-Robin, J.-C., Pujol, S., Bauer, C., Jennings, D., Fennessy, F., Sonka, M., Buatti, J., Aylward, S., Miller, J. V., Pieper, S., \& Kikinis, R. (2012). 3D Slicer as an Image Computing Platform for the Quantitative Imaging Network. Magnetic Resonance Imaging, $\quad 30(9)$, $1323-1341$. https://doi.org/10.1016/j.mri.2012.05.001

Fleiss, J. L. (1971). Measuring nominal scale agreement among many raters. Psychological Bulletin, 76(5), 378-382. https://doi.org/10.1037/h0031619

Franck, C., Lammertyn, J., Ho, Q. T., Verboven, P., Verlinden, B., \& Nicolaï, B. M. (2007). Browning 481 disorders in pear fruit. Postharvest Biology and Technology, 43(1), 1-13. https://doi.org/10.1016/j.postharvbio.2006.08.008

Goodfellow, I., Bengio, Y., \& Courville, A. (2016). Deep learning. The MIT Press.

Graves, M., Smith, A., \& Batchelor, B. (1998). Approaches to foreign body detection in foods. Trends in Food Science \& Technology, 9(1), 21-27. https://doi.org/10.1016/S0924-2244(97)00003-4

Han, D., Tu, R., Lu, C., Liu, X., \& Wen, Z. (2006). Nondestructive detection of brown core in the Chinese pear 'Yali' by transmission visible-NIR spectroscopy. Food Control, 17(8), 604-608. https://doi.org/10.1016/J.FOODCONT.2005.03.006

Herremans, E., Melado-Herreros, A., Defraeye, T., Verlinden, B., Hertog, M., Verboven, P., Val, J., Fernández-Valle, M. E., Bongaers, E., Estrade, P., Wevers, M., Barreiro, P., \& Nicolaï, B. M. (2014). Comparison of X-ray CT and MRI of watercore disorder of different apple cultivars. Postharvest Biology and Technology. https://doi.org/10.1016/j.postharvbio.2013.08.008

Herremans, E., Verboven, P., Bongaers, E., Estrade, P., Verlinden, B. E., Wevers, M., Hertog, M. L. A. T. M., \& Nicolai, B. M. (2013). Characterisation of "Braeburn" browning disorder by means of X-ray micro-CT. Postharvest Biology and Technology. https://doi.org/10.1016/j.postharvbio.2012.08.008 
Huang, Y., Lu, R., \& Chen, K. (2020). Detection of internal defect of apples by a multichannel Vis/NIR spectroscopic system. Postharvest Biology and Technology, 161, 111065. https://doi.org/10.1016/J.POSTHARVBIO.2019.111065

Ioffe, S., \& Szegedy, C. (2015). Batch Normalization: Accelerating Deep Network Training by Reducing Internal Covariate Shift. https://arxiv.org/abs/1502.03167v3

Jaeger, S. R., Antunez, L., Ares, G., Johnston, J. W., Hall, M., \& Roger Harker, F. (2016). Consumers' visual attention to fruit defects and disorders: A case study with apple images. POSTHARVEST $\begin{array}{llll}\text { BIOLOGY AND } & \text { TECHNOLOGY, } & 116, & 36-44 .\end{array}$ https://doi.org/10.1016/j.postharvbio.2015.12.015

Janssens, E., Alves Pereira, L. F., De Beenhouwer, J., Tsang, I. R., Van Dael, M., Verboven, P., Nicolaï, B., \& Sijbers, J. (2016). Fast inline inspection by Neural Network Based Filtered Backprojection: Application to apple inspection. Case Studies in Nondestructive Testing and Evaluation, 6, 14-20. https://doi.org/10.1016/j.csndt.2016.03.003

Janssens, E., De Beenhouwer, J., Van Dael, M., De Schryver, T., Van Hoorebeke, L., Verboven, P., Nicolai, B., \& Sijbers, J. (2018). Neural network Hilbert transform based filtered backprojection for fast inline x-ray inspection. Measurement Science and Technology, 29(3). https://doi.org/10.1088/1361-6501/aa9de3

Khatiwada, B. P., Subedi, P. P., Hayes, C., Carlos, L. C. C., \& Walsh, K. B. (2016). Assessment of internal flesh browning in intact apple using visible-short wave near infrared spectroscopy. Postharvest Biology and Technology, 120, 103-111. https://doi.org/10.1016/J.POSTHARVBIO.2016.06.001

Kingma, D. P., \& Ba, J. (2014). Adam: A Method for Stochastic Optimization. https://arxiv.org/abs/1412.6980

Kotwaliwale, N., Singh, K., Kalne, A., Jha, S. N., Seth, N., \& Kar, A. (2014). X-ray imaging methods for internal quality evaluation of agricultural produce. Journal of Food Science and Technology. https://doi.org/10.1007/s13197-011-0485-y

Kutner, M. H., Nachtsheim, Christopher J., Neter, John, \& Li, William. (2005). Applied Linear Statistical Models. McGraw-Hill Irwin. https://books.google.be/books?id=0xqCAAAACAAJ 
Lammertyn, J., Aerts, M., Verlinden, B. E., Schotsmans, W., \& Nicolaï, B. M. (2000). Logistic regression analysis of factors influencing core breakdown in "Conference" pears. Postharvest Biology and Technology, 20(1), 25-37. https://doi.org/10.1016/S0925-5214(00)00114-9

Lammertyn, J., Dresselaers, T., Van Hecke, P., Jancsók, P., Wevers, M., \& Nicolaï, B. M. (2003a). Analysis of the time course of core breakdown in "Conference" pears by means of MRI and Xray CT. Postharvest Biology and Technology, 29(1), 19-28. https://doi.org/10.1016/S0925$5214(02) 00212-0$

Lammertyn, J., Dresselaers, T., Van Hecke, P., Jancsók, P., Wevers, M., \& Nicolaï, B. M. (2003b). MRI and X-ray CT study of spatial distribution of core breakdown in "Conference" pears. Magnetic Resonance Imaging, 21(7), 805-815. https://doi.org/10.1016/S0730$725 \mathrm{X}(03) 00105-\mathrm{X}$

Landis, J. R., \& Koch, G. G. (1977). The Measurement of Observer Agreement for Categorical Data. Biometrics, 33(1), 159-174. JSTOR. https://doi.org/10.2307/2529310

LeCun, Y., Bengio, Y., \& Hinton, G. (2015). Deep learning. Nature, 521(7553), 436-444.

Litjens, G., Kooi, T., Bejnordi, B. E., Setio, A. A. A., Ciompi, F., Ghafoorian, M., Van Der Laak, J. A., Van Ginneken, B., \& Sánchez, C. I. (2017). A survey on deep learning in medical image analysis. Medical Image Analysis, 42, 60-88.

MATLAB. (2019a). Image Processing Toolbox. The MathWorks Inc.

MATLAB. (2019b). MATLAB. The MathWorks Inc.

Mercier, S., Villeneuve, S., Mondor, M., \& Uysal, I. (2017). Time-Temperature Management Along the Food Cold Chain: A Review of Recent Developments. Comprehensive Reviews in Food Science and Food Safety, 16(4), 647-667. https://doi.org/10.1111/1541-4337.12269

Metz, C. E. (1978). Basic principles of ROC analysis. Seminars in Nuclear Medicine, 8(4), 283-298. https://doi.org/10.1016/S0001-2998(78)80014-2

Milesial. (2019). Pytorch-UNet. In GitHub repository. GitHub. https://github.com/milesial/PytorchUNet

Nair, V., \& Hinton, G. E. (2010). Rectified Linear Units Improve Restricted Boltzmann Machines. 8. 
Nicolaï, B. M., Defraeye, T., De Ketelaere, B., Herremans, E., Hertog, M. L. A. T. M., Saeys, W., Torricelli, A., Vandendriessche, T., \& Verboven, P. (2014). Nondestructive Measurement of Fruit and Vegetable Quality. Annual Review of Food Science and Technology. https://doi.org/10.1146/annurev-food-030713-092410

Nugraha, B., Verboven, P., Janssen, S., Wang, Z., \& Nicolaï, B. M. (2019). Non-destructive porosity mapping of fruit and vegetables using X-ray CT. Postharvest Biology and Technology, 150, 80-88. https://doi.org/10.1016/j.postharvbio.2018.12.016

Otsu, N. (1979). A Threshold Selection Method from Gray-Level Histograms. IEEE Transactions on Systems, Man, and Cybernetics, 9(1), 62-66. https://doi.org/10.1109/TSMC.1979.4310076

Paszke, A., Gross, S., Massa, F., Lerer, A., Bradbury, J., Chanan, G., Killeen, T., Lin, Z., Gimelshein, N., Antiga, L., Desmaison, A., Kopf, A., Yang, E., DeVito, Z., Raison, M., Tejani, A., Chilamkurthy, S., Steiner, B., Fang, L., ... Chintala, S. (2019). PyTorch: An Imperative Style, High-Performance Deep Learning Library. In H. Wallach, H. Larochelle, A. Beygelzimer, F. dltextquotesingle Alché-Buc, E. Fox, \& R. Garnett (Eds.), Advances in Neural Information Processing Systems 32 (pp. 8024-8035). Curran Associates, Inc. http://papers.neurips.cc/paper/9015-pytorch-an-imperative-style-high-performance-deeplearning-library.pdf

Pedreschi, R., Franck, C., Lammertyn, J., Erban, A., Kopka, J., Hertog, M., Verlinden, B., \& Nicolaï, B. (2009). Metabolic profiling of "Conference" pears under low oxygen stress. Postharvest Biology and Technology, 51(2), 123-130. https://doi.org/10.1016/j.postharvbio.2008.05.019

Pereira, L. F. A., Janssens, E., Cavalcanti, G. D. C., Tsang, I. R., Van Dael, M., Verboven, P., Nicolai, B., \& Sijbers, J. (2017). Inline discrete tomography system: Application to agricultural product inspection. Computers and Electronics in Agriculture, 138, 117-126. https://doi.org/10.1016/J.COMPAG.2017.04.010

Rezatofighi, H., Tsoi, N., Gwak, J., Sadeghian, A., Reid, I., \& Savarese, S. (2019). Generalized Intersection over Union. The IEEE Conference on Computer Vision and Pattern Recognition (CVPR). 

Segmentation. http://arxiv.org/abs/1505.04597

581

Ruder, S. (2017). An Overview of Multi-Task Learning in Deep Neural Networks. ArXiv:1706.05098 [Cs, Stat]. http://arxiv.org/abs/1706.05098

Shen, D., Wu, G., \& Suk, H.-I. (2017). Deep Learning in Medical Image Analysis. Annual Review of Biomedical Engineering, 19(1), 221-248. https://doi.org/10.1146/annurev-bioeng-071516044442

Sudre, C. H., Li, W., Vercauteren, T., Ourselin, S., \& Jorge Cardoso, M. (2017). Generalised Dice Overlap as a Deep Learning Loss Function for Highly Unbalanced Segmentations. Lecture Notes in Computer Science, 240-248. https://doi.org/10.1007/978-3-319-67558-9_28

Tharwat, A. (2016). Linear vs. Quadratic discriminant analysis classifier: A tutorial. International Journal of Applied Pattern Recognition, 3(2), 145-180.

van Dael, M., Verboven, P., Dhaene, J., Van Hoorebeke, L., Sijbers, J., \& Nicolai, B. (2017). Multisensor X-ray inspection of internal defects in horticultural products. Postharvest Biology and Technology, 128, 33-43. https://doi.org/10.1016/j.postharvbio.2017.02.002

van Dael, Verboven, P., Zanella, A., Sijbers, J., \& Nicolai, B. (2018). Combination of shape and X-ray inspection for apple internal quality control: In silico analysis of the methodology based on Xray computed tomography. Postharvest Biology and Technology, May, 0-1. https://doi.org/10.1016/j.postharvbio.2018.05.020

Van De Looverbosch, T., Bhuiyan, Md. H. R., Verboven, P., Dierick, M., Van Loo, D., De Beenbouwer, J., Sijbers, J., \& Nicolaï, B. (2020). Nondestructive internal quality inspection of pear fruit by X-ray CT using machine learning. Food Control, 113.

VCBT. (2017). Bewaarcondities Appel en Peer. http://vcbt.be/bewaarcondities_appel_en_peer/

Veltman, R. H., Lenthéric, I., Van Der Plas, L. H. W., \& Peppelenbos, H. W. (2003). Internal browning in pear fruit (Pyrus communis L. cv Conference) may be a result of a limited availability of energy and antioxidants. Postharvest Biology and Technology, 28(2), 295-302. https://doi.org/10.1016/S0925-5214(02)00198-9 

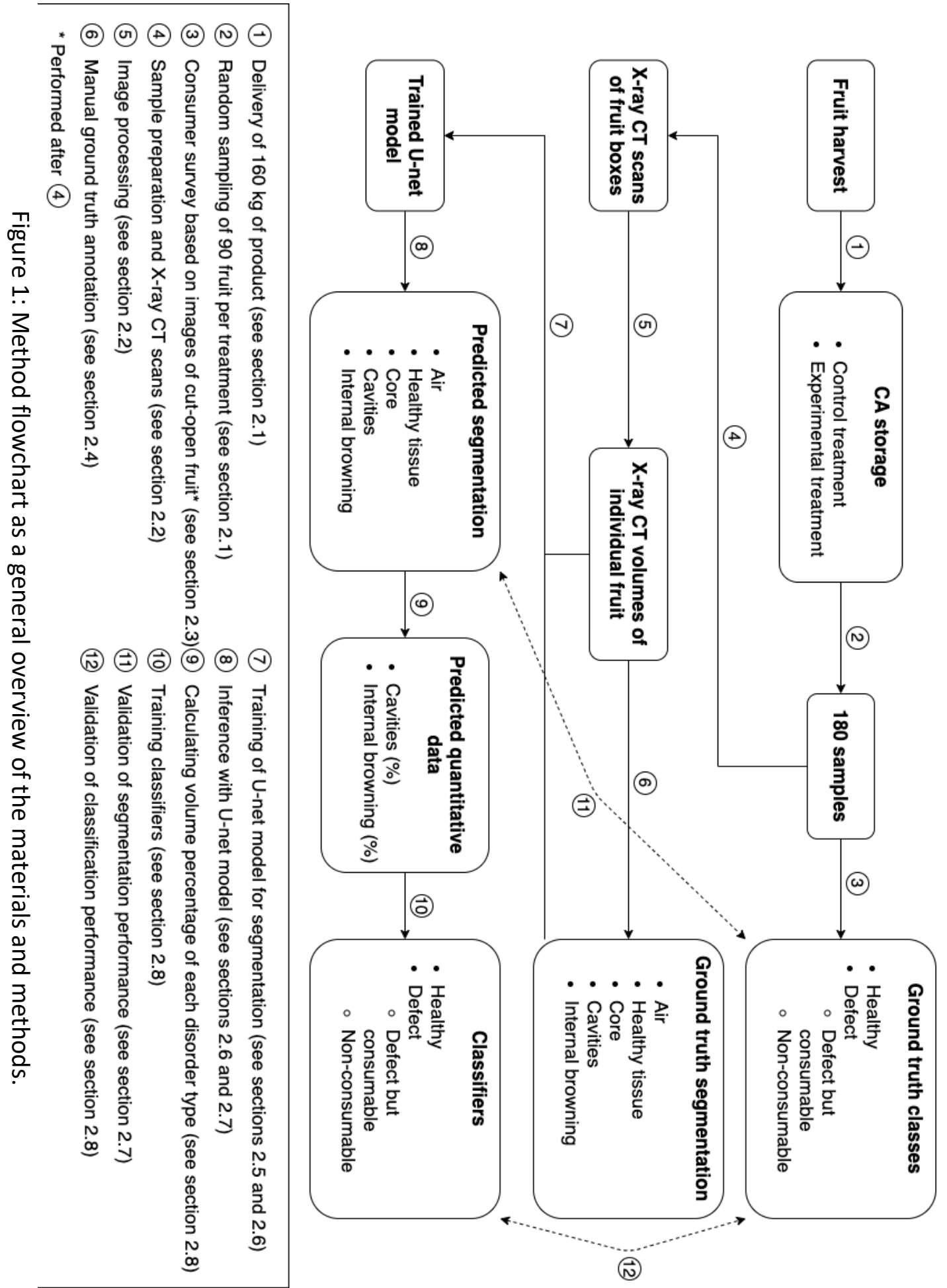


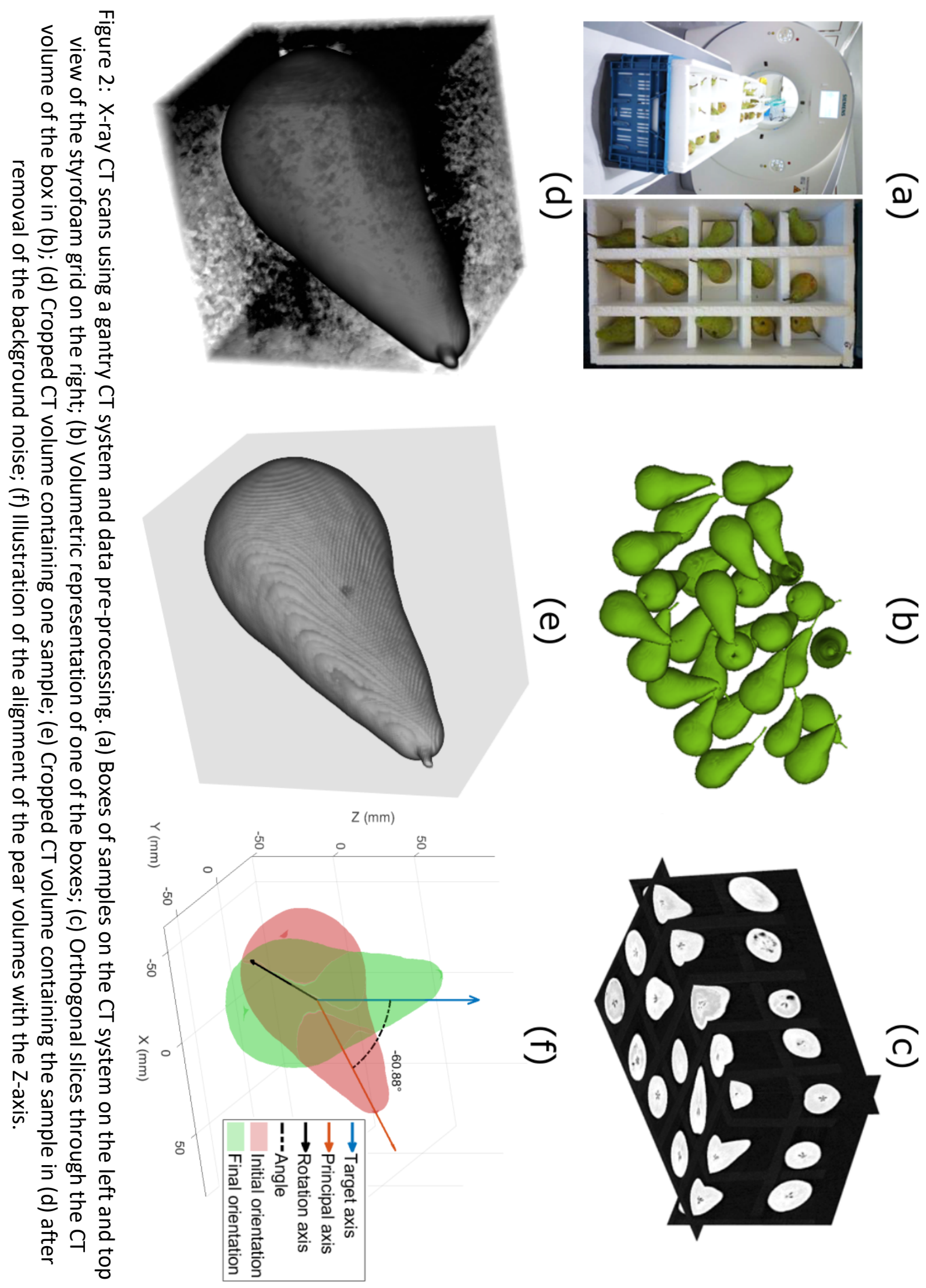




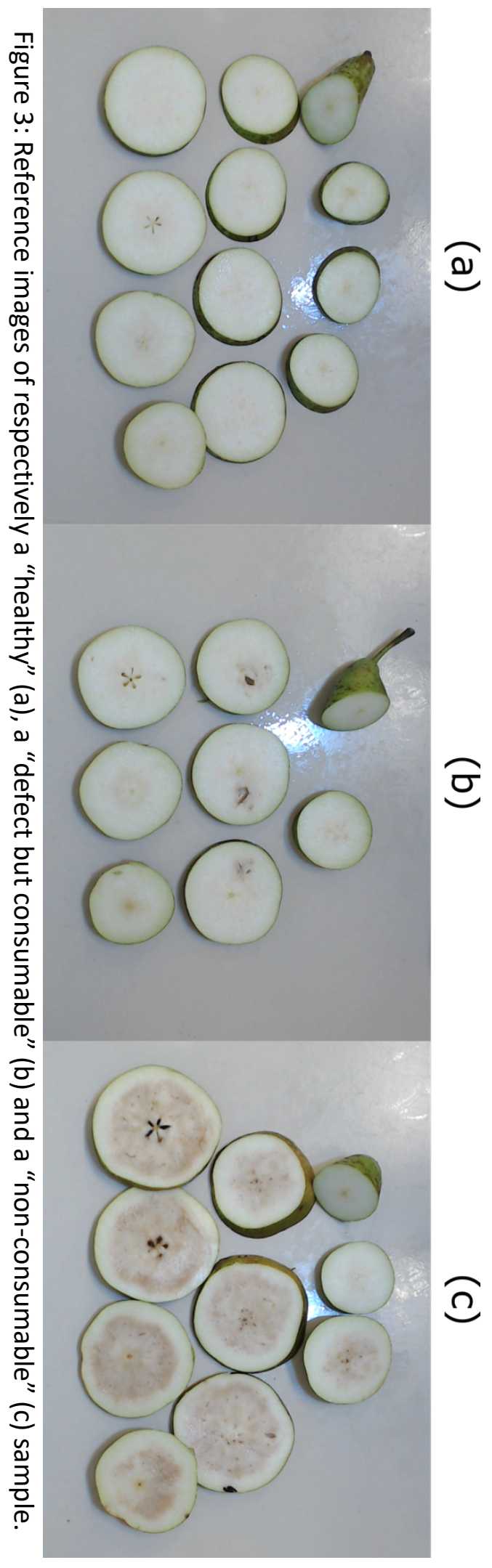


(a)
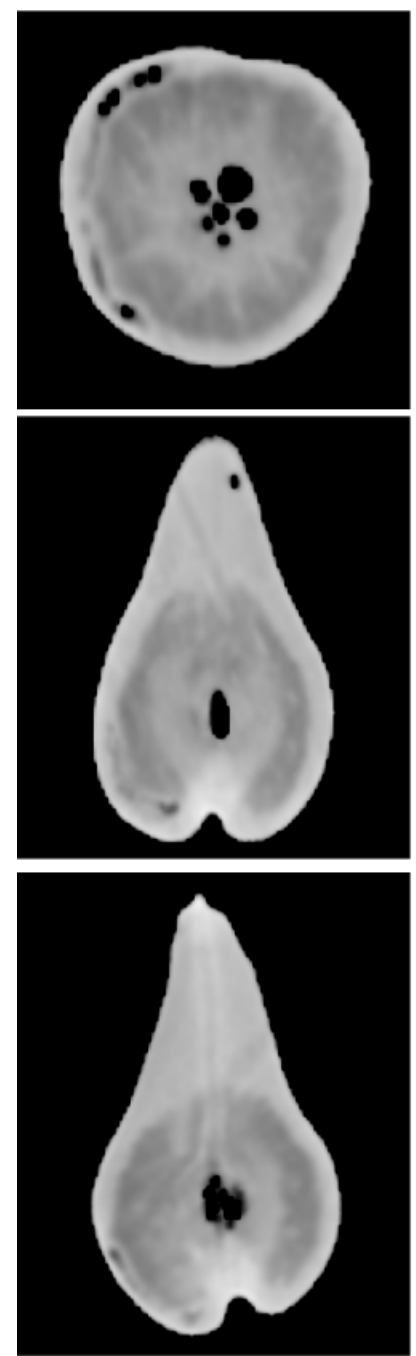

\section{External}

(b)
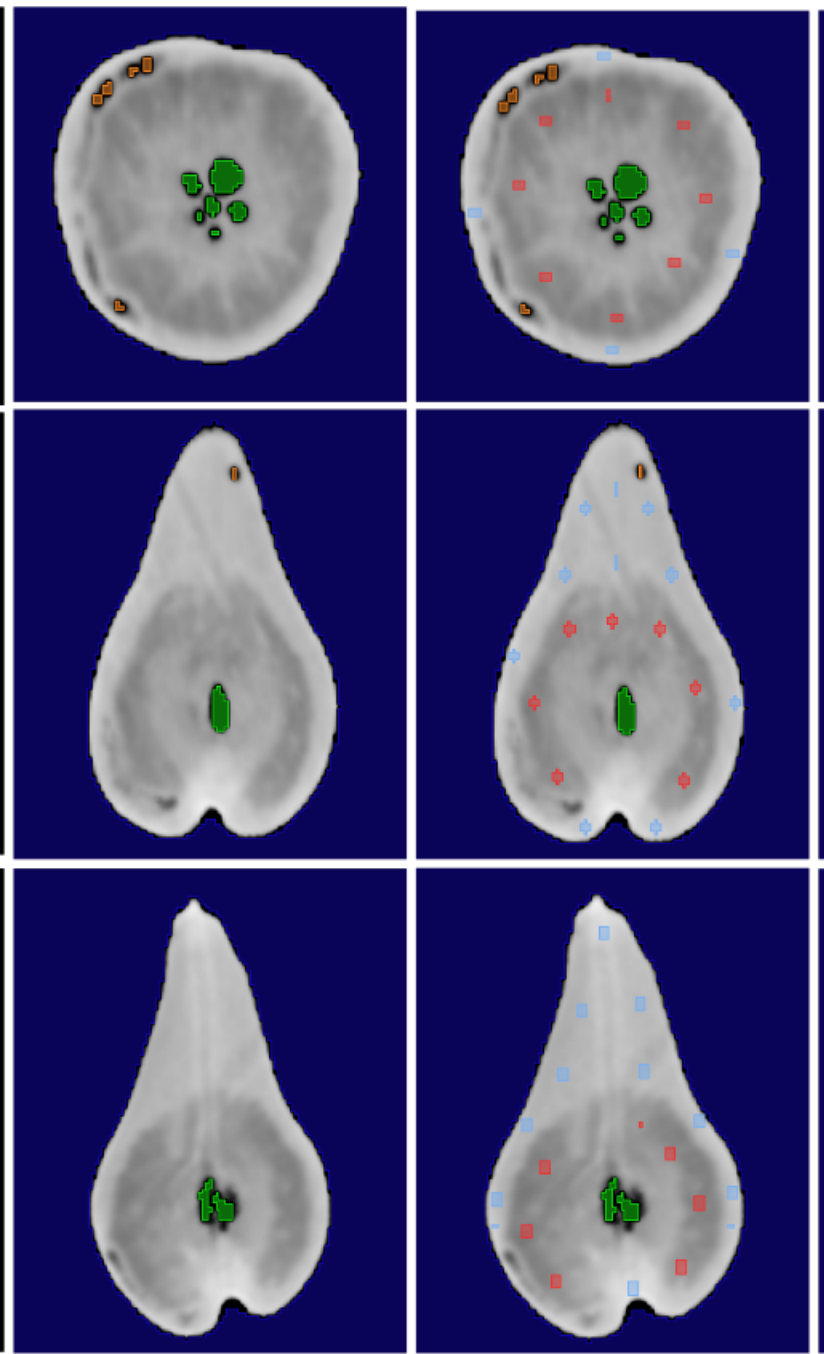
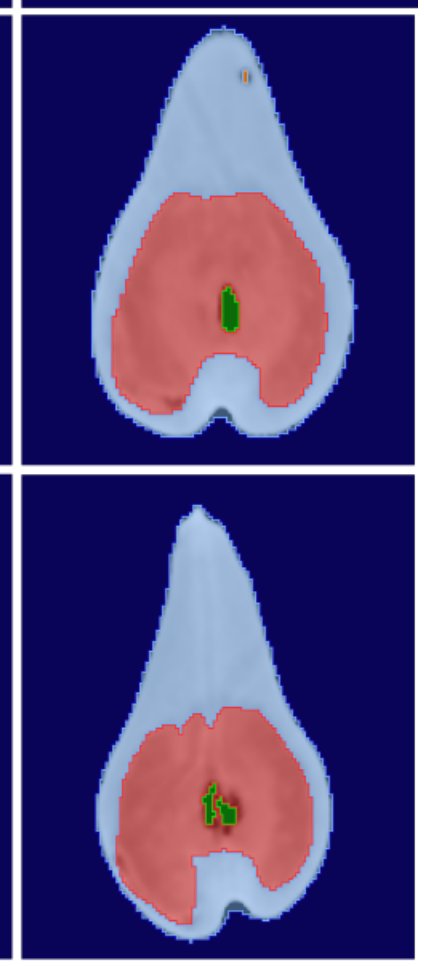

Browning

(d)

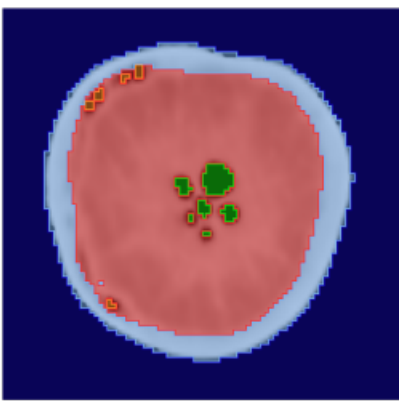

Cavity

Figure 4: Steps in the manual annotation of CT volumes. (a) Initial CT volume after background noise removal with global threshold; (b) CT volume with external (dark blue), core (green) and cavity (orange) voxel indicated; (c) Addition of seed points of healthy tissue (light blue) and browning (red) voxels for subsequent region growing step; (d) Final result of the annotation procedure with healthy tissue (light blue) and browning (red) voxels resulting from region growing using the seeds in (c). 


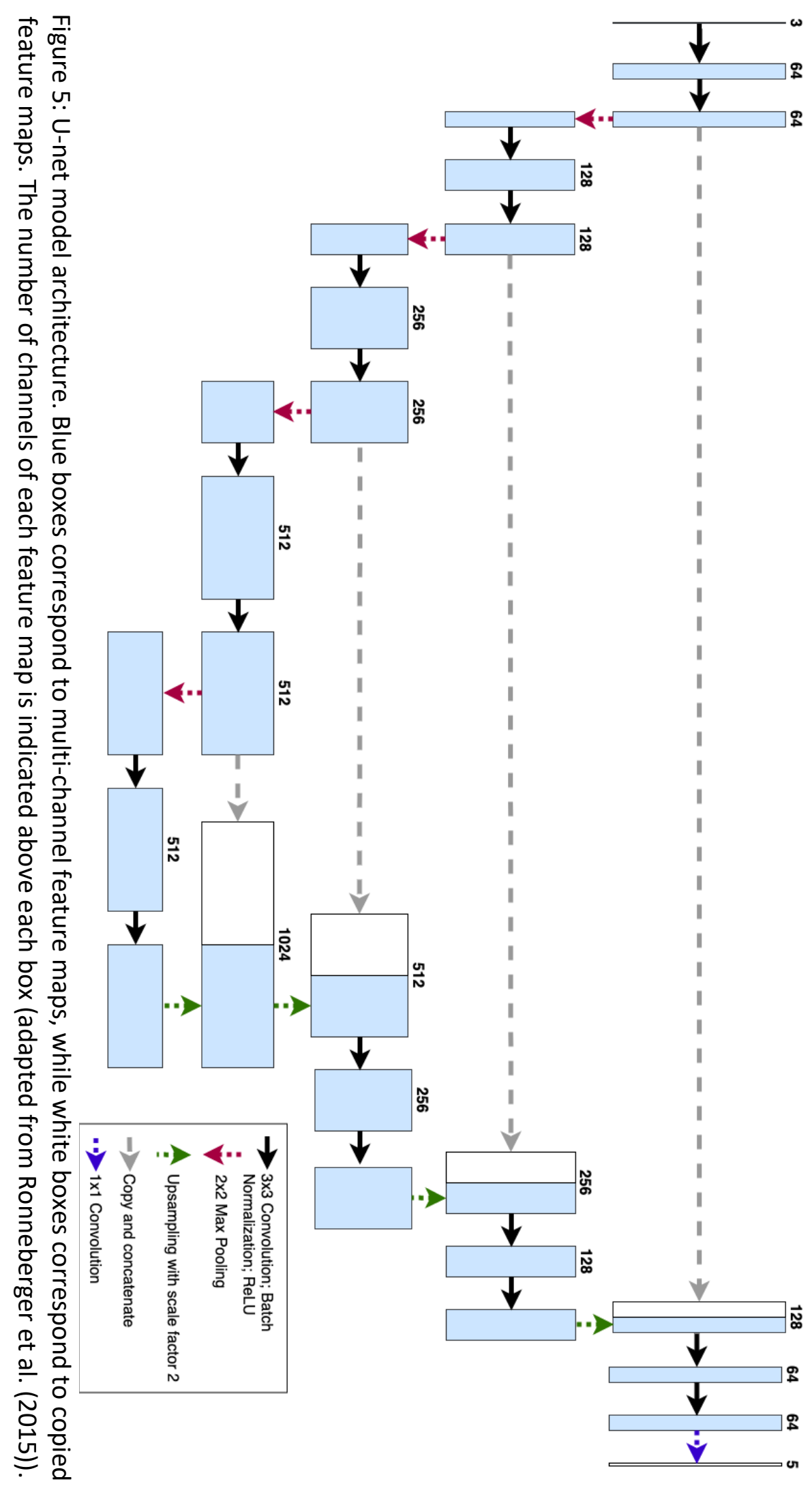




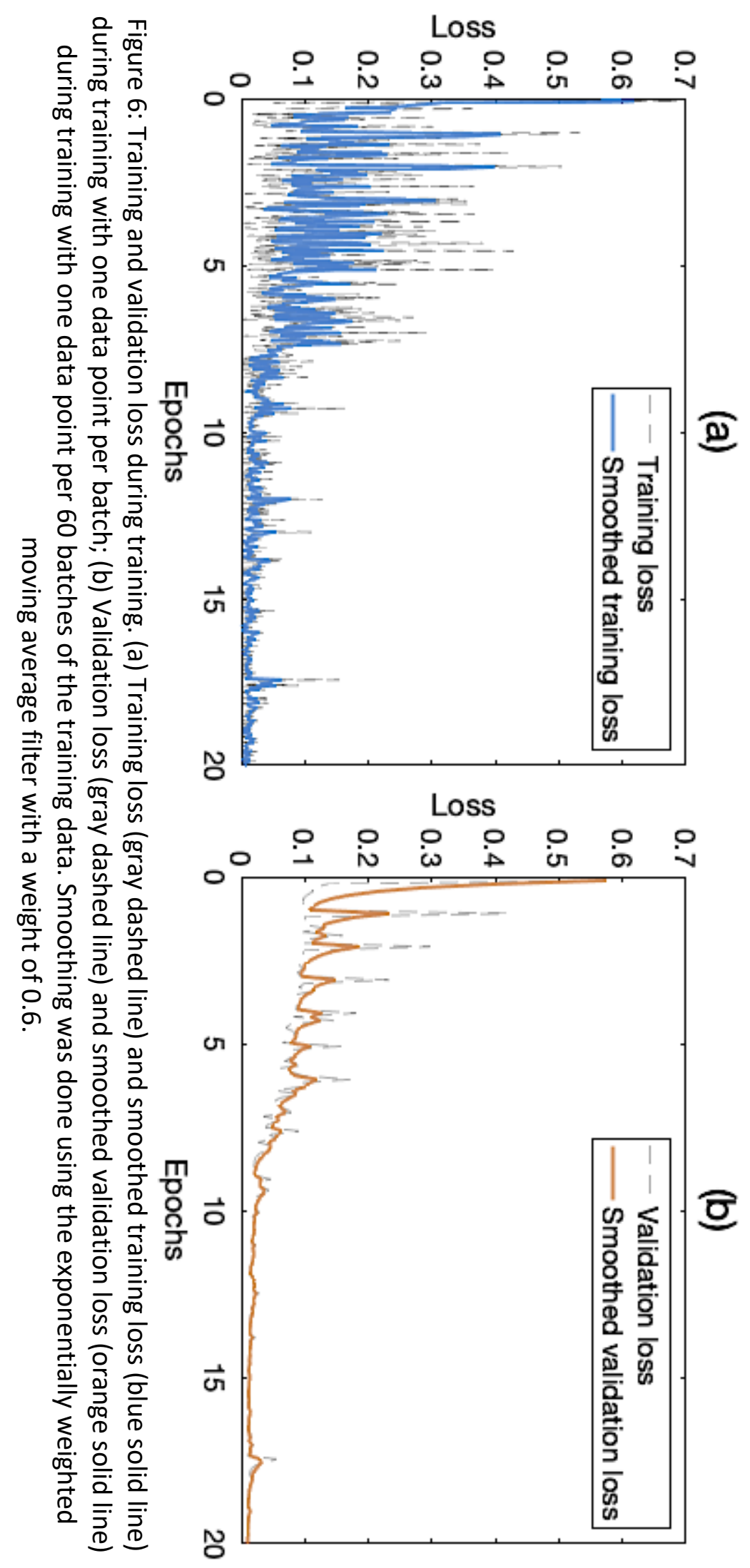


(a)
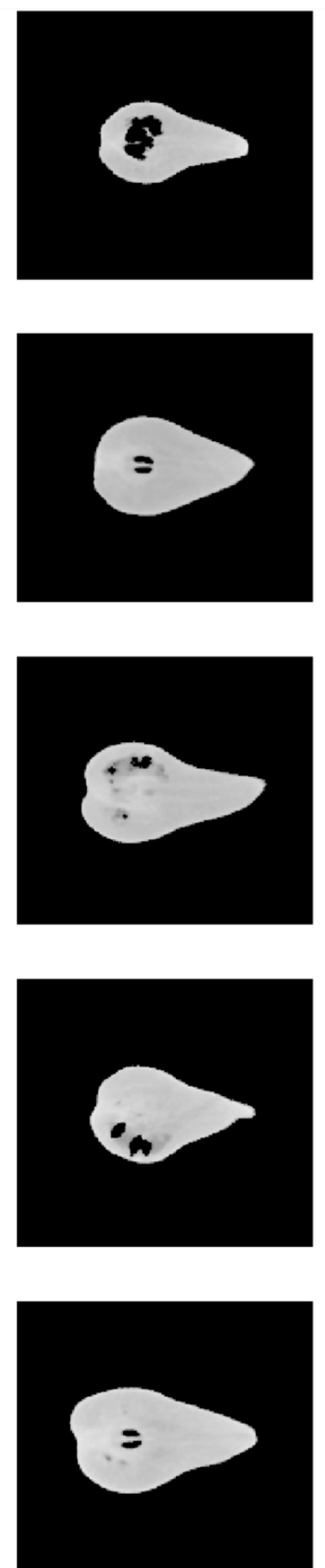

\section{External}
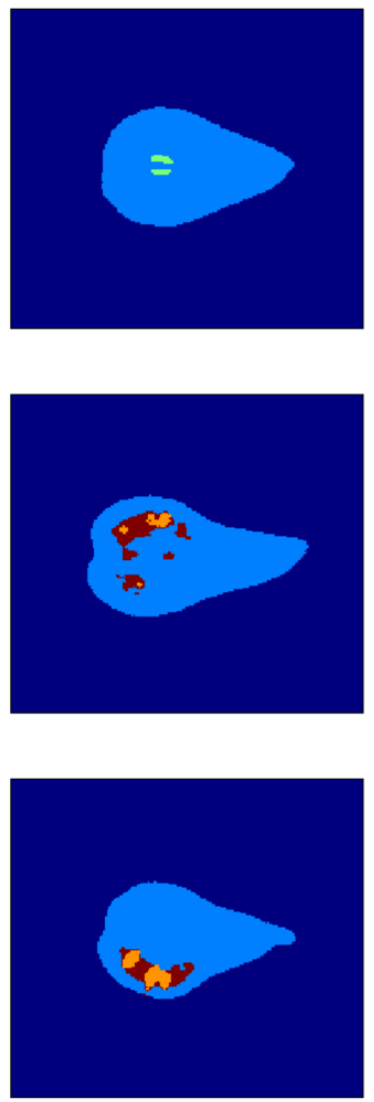

(b)
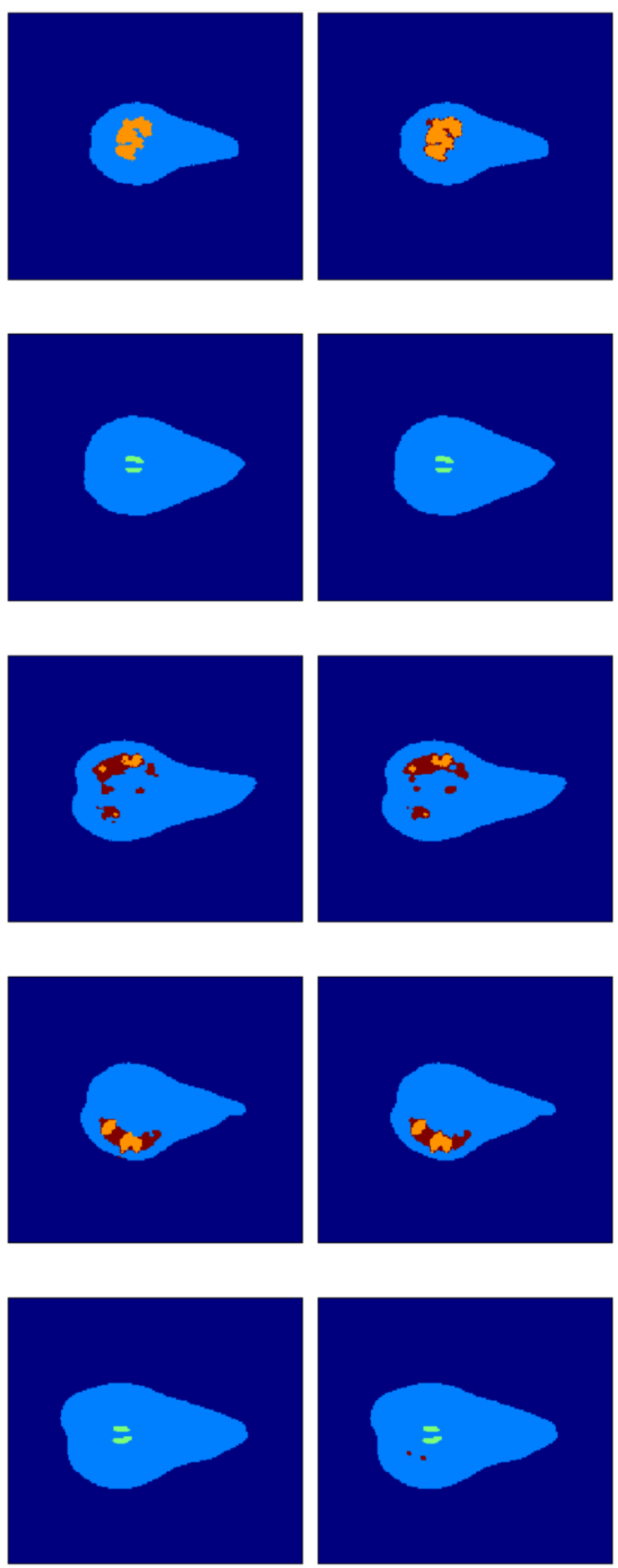

Tissue

(c)

Core (d)
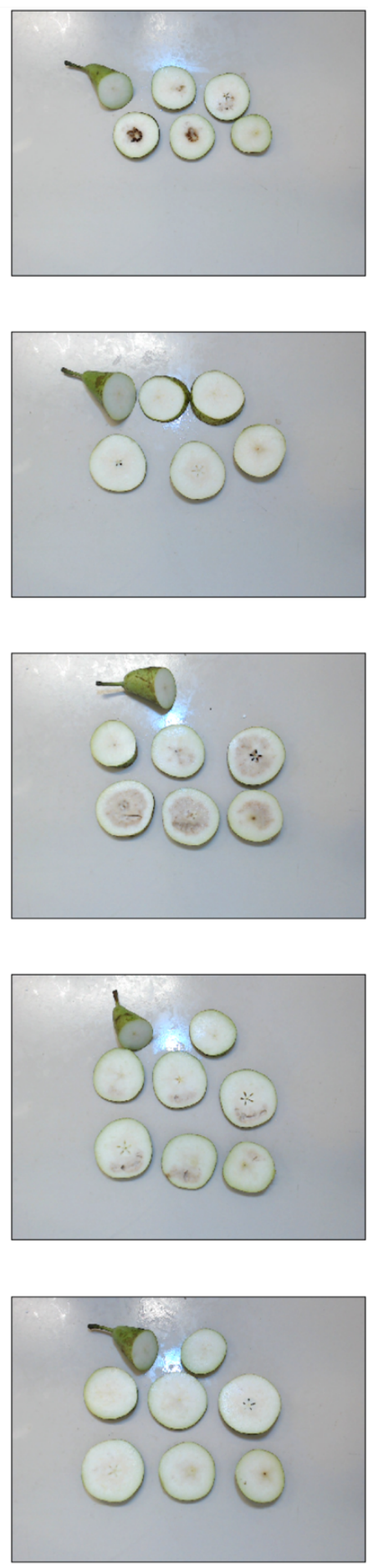

Cavity
Browning

Figure 7: Examples of visual segmentation results on the test set. Each row represents another pear. (a) XY slice of the original CT scan; (b) the corresponding ground truth segmentation; (c) the corresponding predicted segmentation; (d) the reference image of the corresponding pear. 


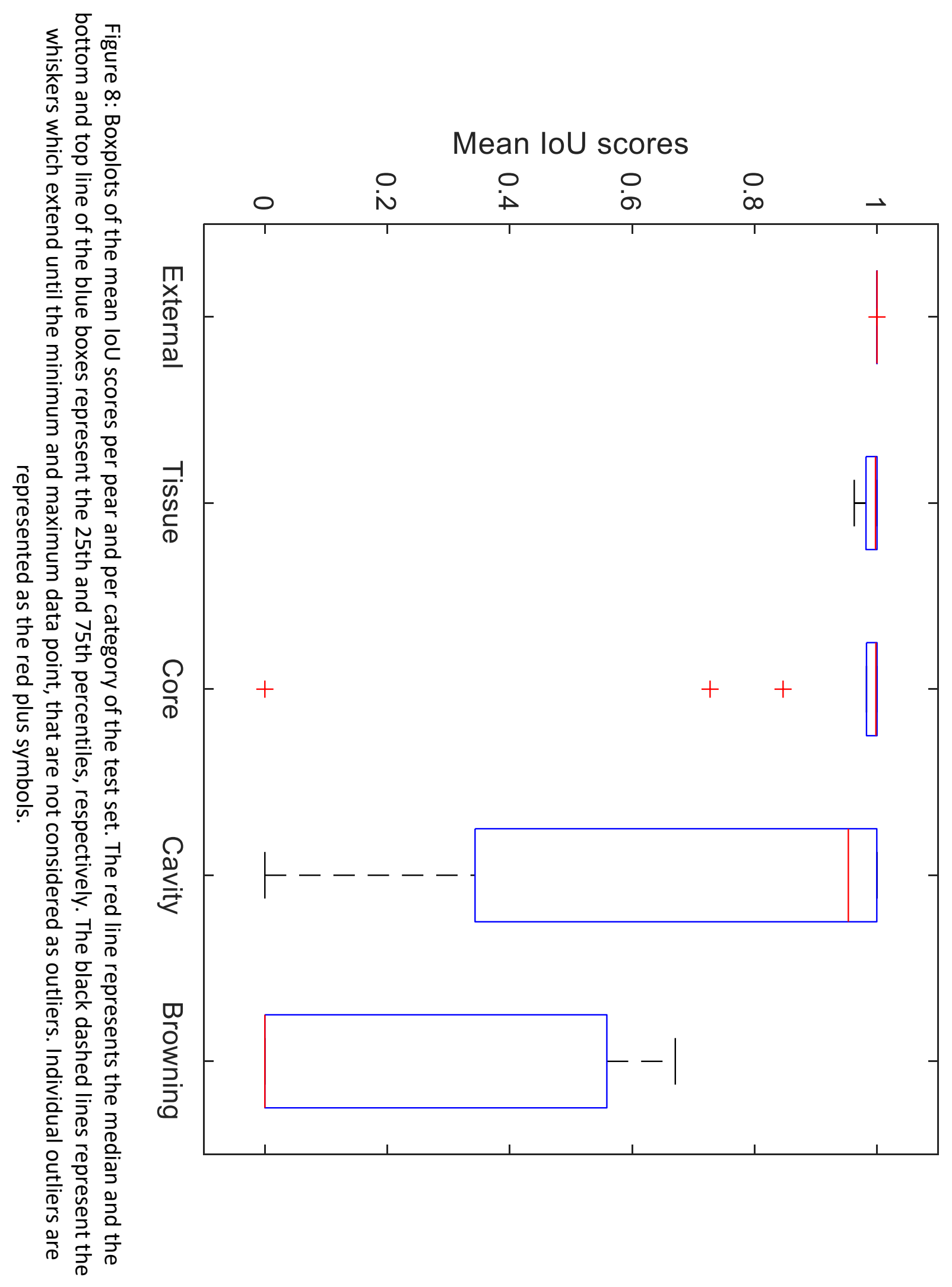




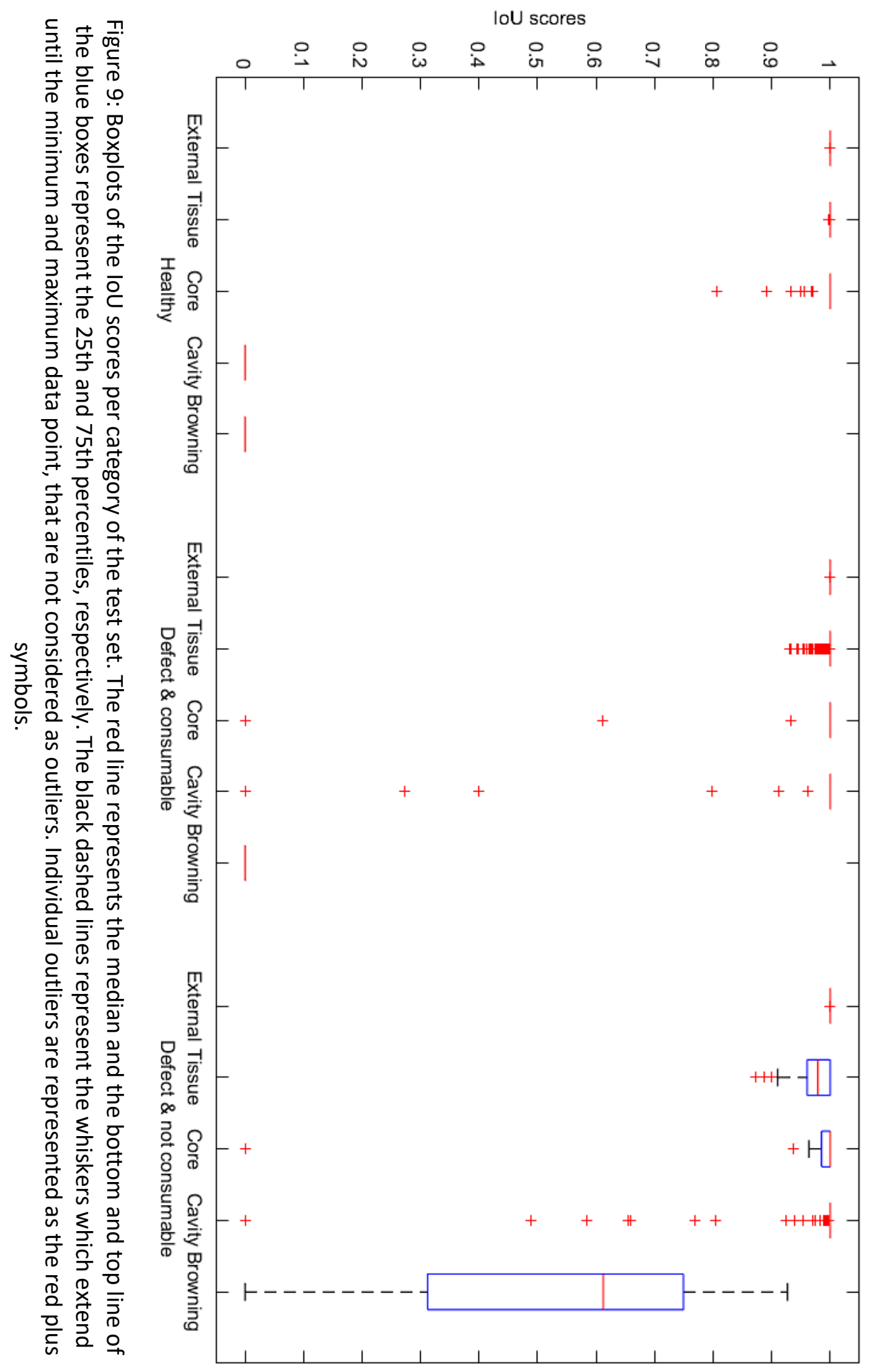




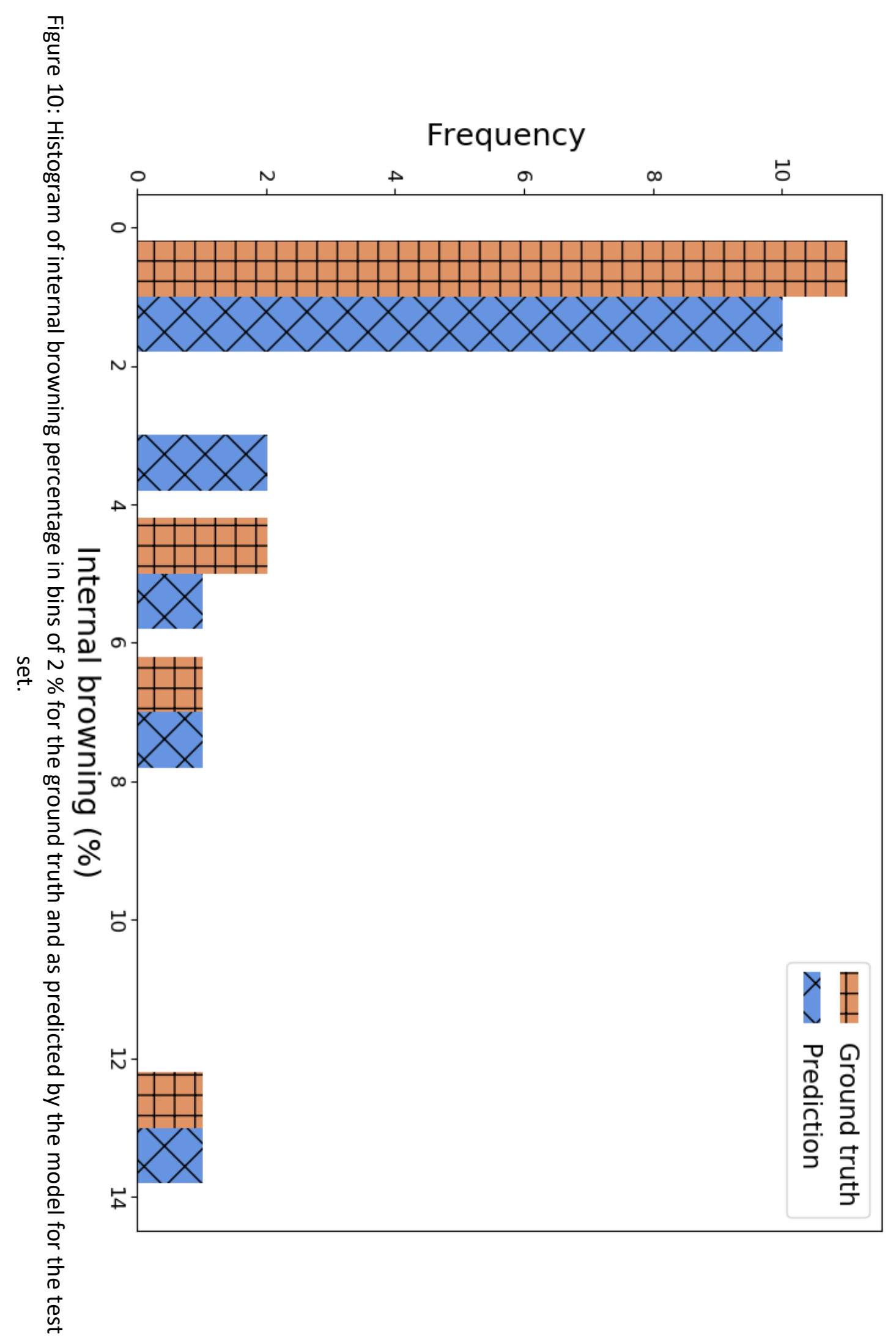




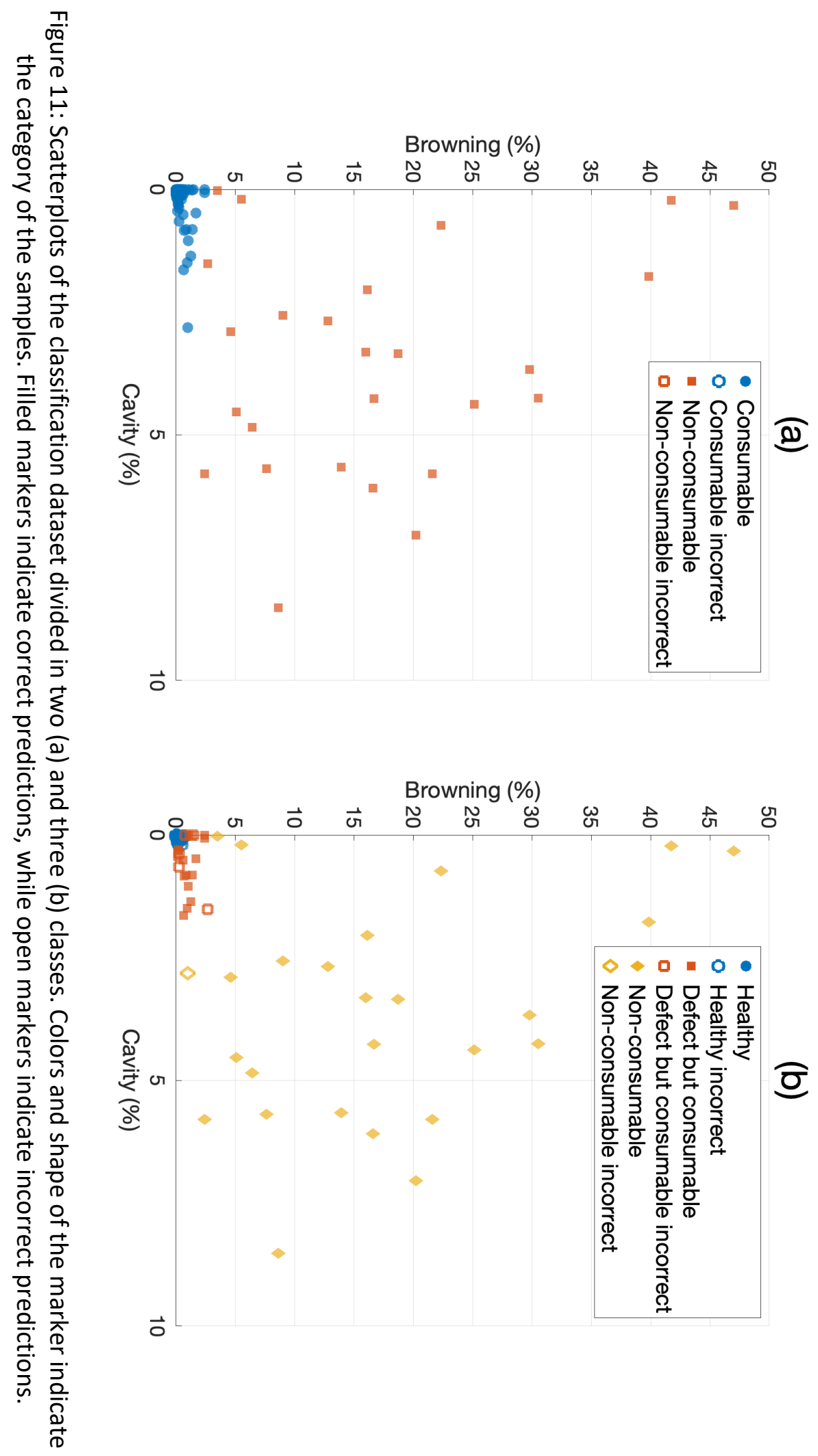




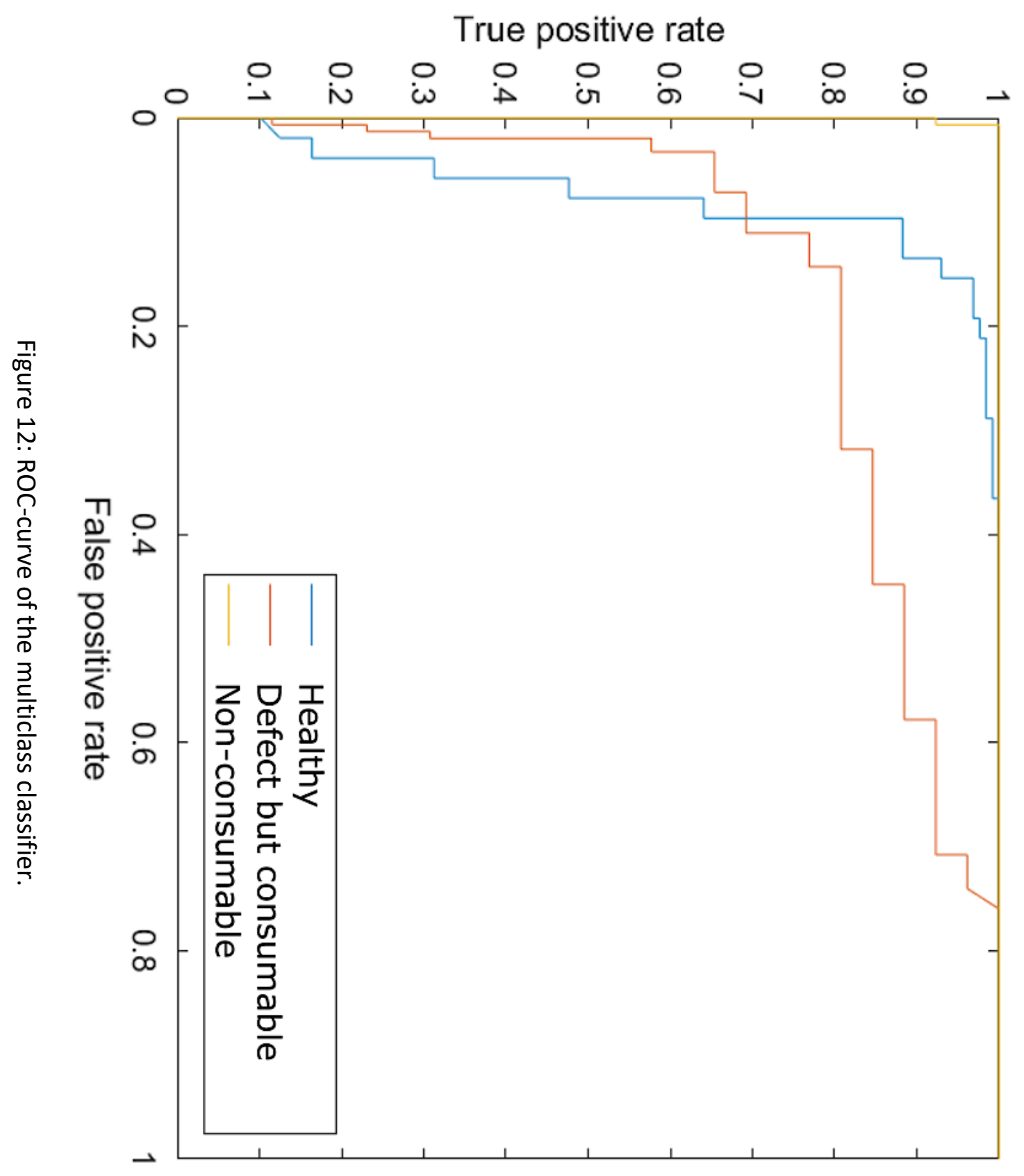




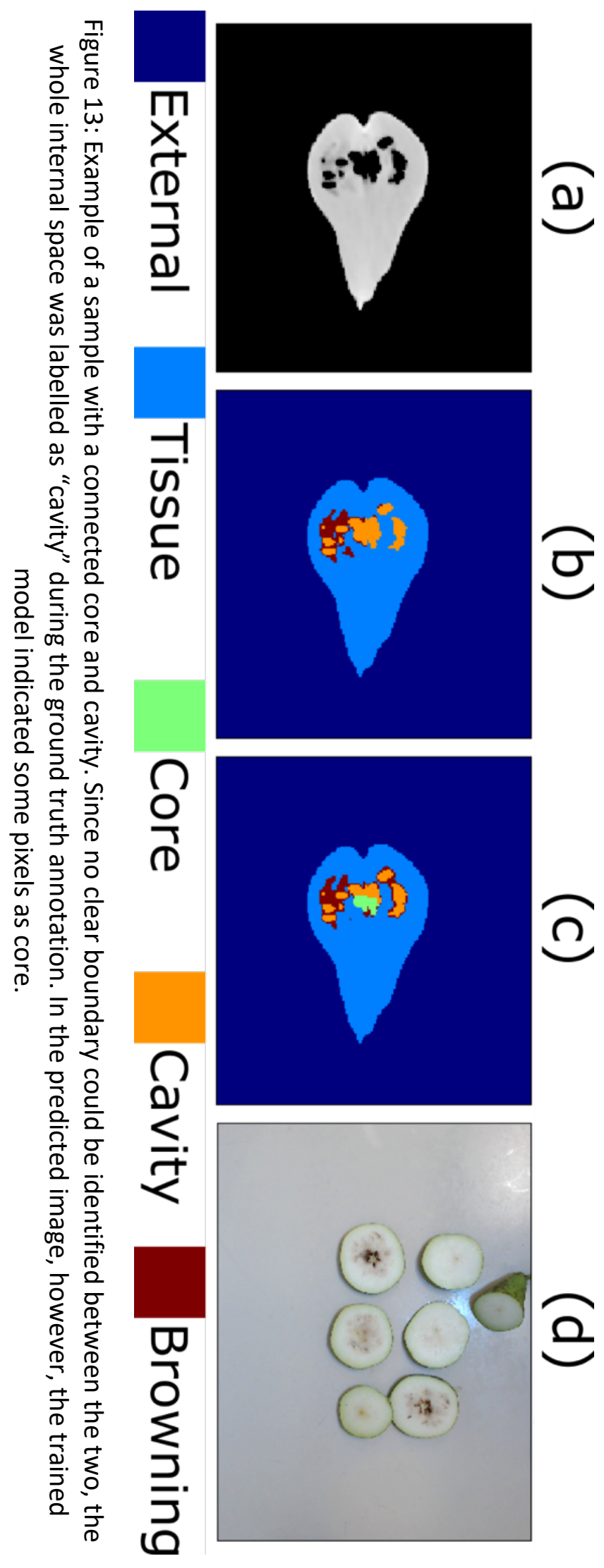




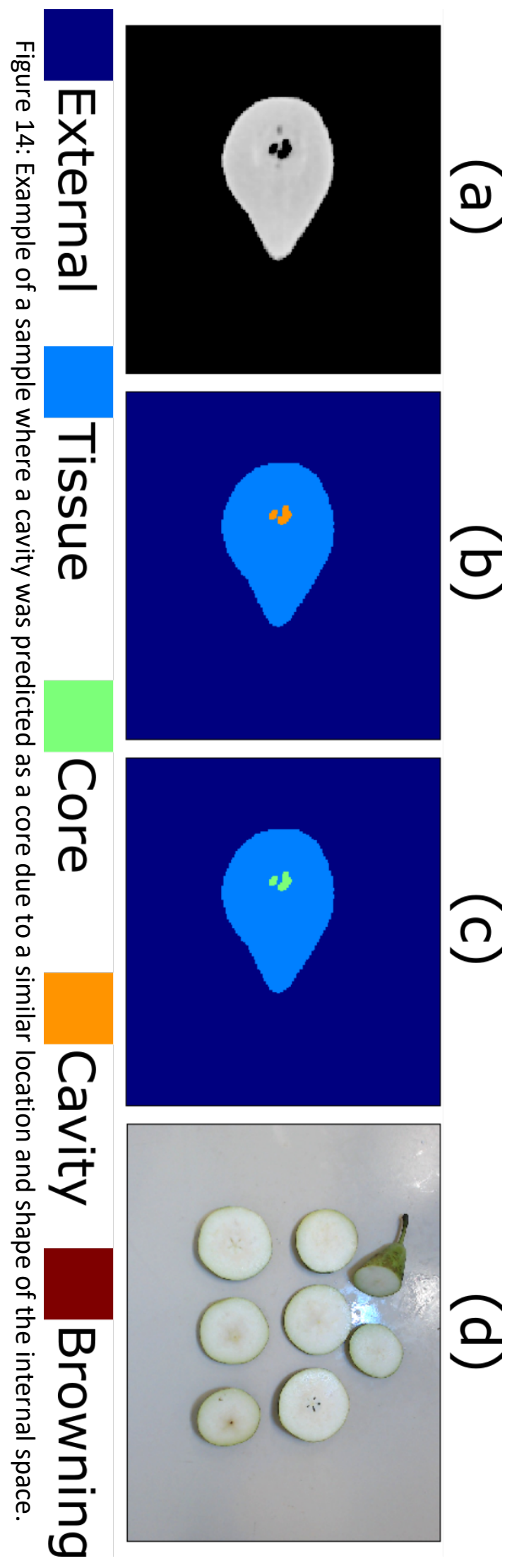




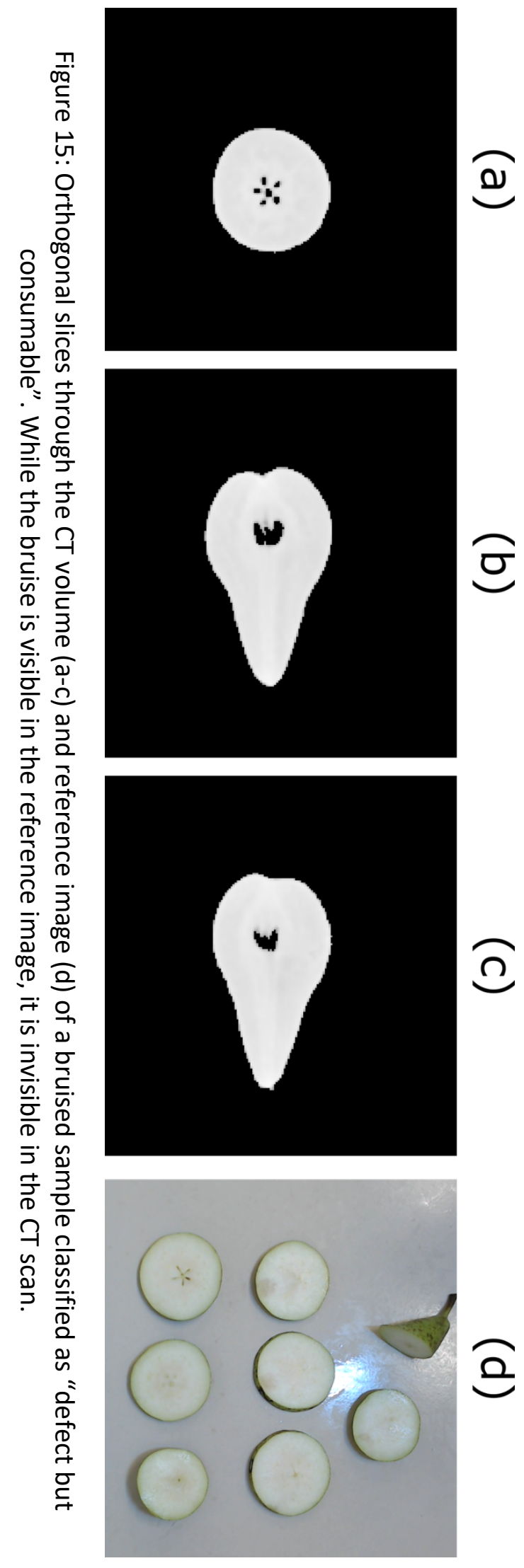


Table 1: Confusion matrix of the binary classifier.

\begin{tabular}{llll}
\hline & & Predicted & \\
\cline { 3 - 4 } & & Consumable & Non-consumable \\
\hline \multirow{2}{*}{ Ground truth } & Consumable & $100.0 \%$ & $0.0 \%$ \\
& Non-consumable & $4.0 \%$ & $96.0 \%$ \\
\hline & Overall accuracy & $99.4 \%$ & \\
\hline
\end{tabular}


Table 2: Confusion matrix of the multiclass classifier.

\begin{tabular}{lllll}
\hline \multirow{2}{*}{ Ground truth } & Healthy & $97.0 \%$ & $3.0 \%$ & \\
\cline { 3 - 5 } & Healthy & Defect but consumable & Non-consumable \\
\hline & $\begin{array}{l}\text { Defect but } \\
\text { consumable }\end{array}$ & $31.0 \%$ & $65.0 \%$ & $0.0 \%$ \\
& Non-consumable & $0.0 \%$ & $4.0 \%$ & $4.0 \%$ \\
\hline & Overall accuracy & $92.2 \%$ & & $96.0 \%$ \\
\hline
\end{tabular}

